TRANSACTIONS OF THE

AMERICAN MATHEMATICAL SOCIETY

Volume 351, Number 4, April 1999, Pages 1663-1690

S 0002-9947(99)02098-X

\title{
VERTEX OPERATORS FOR TWISTED QUANTUM AFFINE ALGEBRAS
}

\author{
NAIHUAN JING AND KAILASH C. MISRA
}

\begin{abstract}
We construct explicitly the $q$-vertex operators (intertwining operators) for the level one modules $V\left(\Lambda_{i}\right)$ of the classical quantum affine algebras of twisted types using interacting bosons, where $i=0,1$ for $A_{2 n-1}^{(2)}(n \geq 3)$, $i=0$ for $D_{4}^{(3)}, i=0, n$ for $D_{n+1}^{(2)}(n \geq 2)$, and $i=n$ for $A_{2 n}^{(2)}(n \geq 1)$. A perfect crystal graph for $D_{4}^{(3)}$ is constructed as a by-product.
\end{abstract}

\section{INTRODUCTION}

In a major step toward understanding $q$-conformal field theory [5], Frenkel and Reshetikhin introduced the $q$-Knizhnik-Zamoldchikov equation associated with the quantum affine algebras. The main theoretical tool they used was that of certain intertwining operators called $q$-vertex operators between two types of representations of quantum affine algebras. They showed that the matrix coefficients of these vertex operators will give the solutions of the $q$-KZ equations.

On the other hand, in a series of works by the Kyoto School and their collaborators elsewhere (cf. the monograph [13] for details) we see that the very same vertex operators take the role of corner transfer matrices in vertex models in statistical mechanics. The subsequent analysis enabled them to provide rigorous mathematical theory to explain the appearance of Baxter's method of the corner transfer matrix. The space of physical states is understood as a tensor product of highest weight representations of quantum affine algebras [1]. In level one case since the explicit realization of the underlying quantum affine algebra [4] is available, the method works particularly well. For instance, one feature of their work is that the correlation functions can be computed in an explicit form using the bosonic realizations of the $q$-vertex operators [7].

The program of understanding various vertex models relies upon the explicit knowledge of the $q$-vertex operators associated with quantum affine algebras. So far the explicit realization of level one $q$-vertex operators is carried out for the quantum affine algebras in the cases of $A_{n}^{(1)}[7,14], D_{n}^{(1)}[12]$ and $B_{n}^{(1)}$ [13]. The level two cases of $A_{1}^{(1)}$ were treated in [6] and, as special cases of our general construction, in [13]. Some other cases for reducible modules are also given in [16, 18].

Received by the editors August 30, 1996 and, in revised form, March 11, 1997.

1991 Mathematics Subject Classification. Primary 17B37, 17B67; Secondary 82B23, 81R10, 81R50.

Key words and phrases. Quantum affine algebras, $q$-vertex operators.

The first author is supported in part by NSA grants MDA 904-94-H-2061 and MDA 904-96-10087. The second author is supported in part by NSA grant MDA 904-96-1-0013.

(C)1999 American Mathematical Society 
In this paper we will give explicit construction of level one $q$-vertex operators for twisted quantum affine algebras of types $A_{2 n-1}^{(2)}, D_{n+1}^{(2)}, A_{2 n}^{(2)}$ (including $A_{2}^{(2)}$ ) and $D_{4}^{(3)}$. Our work is based on the explicit realization of level one twisted quantum affine algebras given first in [9] for $\Lambda_{0}$. We generalize the realization to other level one irreducible modules in Section 3.

In section 2 we will review some preliminary results about quantum affine algebras and the Drinfeld realization written in a modified form [11]. Based on [11] we calculate the special level zero finite dimensional representations for twisted quantum affine algebras. We then recall the level one realization of twisted quantum affine algebras [9] and in particular we extend the construction to all other level one modules by the coset method. In the final section we give the explicit construction of the level one $q$-vertex operators for classical twisted quantum affine algebras.

In the previous construction of $q$-vertex operators, the finite dimensional level zero representations are exactly coming from the crystal graphs of the quantum affine algebras. We found that this is no longer true for the twisted cases. In the case of $D_{4}^{(3)}$ we constructed an 8-dimensional representation and its associated perfect crystal graph (cf. (2.11)). As far as we know, this is the first perfect crystal structure for $D_{4}^{(3)}$.

Another novelty is that the $q$-Heisenburg subalgebra in the Drinfeld generators of the level zero modules is no longer diagonalizable. This is due to the fact that the underlying vector space representation may admit a special 1-dimensional subspace, which is used in the perfect crystal graphs for types $D_{n+1}^{(2)}, A_{2 n}^{(2)}$ and $D_{4}^{(3)}$ (cf. Section 2).

\section{Twisted quantum Affine algebras $U_{q}\left(X_{N}^{(r)}\right)$}

Let $\mathbf{g}$ be the finite dimensional simple Lie algebra $\mathbf{s l}(2 n)(n \geq 3)$, $\mathbf{s o}(2 n+2)$ $(n \geq 2), \mathbf{s l}(2 n+1)(n \geq 1)$ or $\mathbf{s o}(8)$. We associate an integer $r$ to the four cases as follows: $r=2,2,2,3$ respectively. We denote the Dynkin diagram by $\Gamma$ and label their simple roots and coroots as

$$
\alpha_{1}^{\prime}, \cdots, \alpha_{N}^{\prime}, \quad h_{1}^{\prime}, \cdots, h_{N}^{\prime}
$$

where $N=2 n-1, n+1,2 n, 4$, respectively, $\alpha_{i} \in \mathbf{h}^{\prime *}$ and $\mathbf{h}^{\prime}=\mathbf{C} h_{1}^{\prime} \oplus \cdots \oplus \mathbf{C} h_{N}^{\prime}$ is the Cartan subalgebra of $\mathbf{g}$. We denote their Chevalley generators by

$$
e_{i}^{\prime}, f_{i}^{\prime}, h_{i}^{\prime}, \quad i=1, \cdots, N
$$

The root and weight lattices are then defined by

$$
\begin{aligned}
Q^{\prime} & =\mathbf{Z} \alpha_{1}^{\prime}+\cdots+\mathbf{Z} \alpha_{N}^{\prime}, \\
P^{\prime} & =\mathbf{Z} \lambda_{1}^{\prime}+\cdots+\mathbf{Z} \lambda_{N}^{\prime}
\end{aligned}
$$

where $\lambda_{i}^{\prime}$ are the fundamental weights of the Lie algebra $\mathbf{g}: \lambda_{i}^{\prime}\left(h_{j}^{\prime}\right)=\delta_{i j}, i, j=$ $1, \cdots, N$.

We let $\langle. \mid$.$\rangle be the nondegenerate invariant bilinear form on \mathbf{g}$ normalized by $\langle\alpha \mid \alpha\rangle=2$, i.e., $\langle x \mid y\rangle=\operatorname{tr}(x y), \frac{1}{2} \operatorname{tr}(x y), \operatorname{tr}(x y), \frac{1}{2} \operatorname{tr}(x y)$ respectively. Then $\left(h_{i}^{\prime} \mid h_{i}^{\prime}\right)=$ 2 for $i=1, \cdots, N$. Since the Lie algebra $\mathbf{g}$ is simply-laced, we can identify the invariant form on $\mathbf{h}^{* *}$ to that of $\mathbf{h}^{\prime}$. 
Let $\sigma$ be a diagram automorphism of order $r=2,3$ for the Lie algebra $\mathbf{g}$ such that

$$
\begin{aligned}
& \sigma\left(h_{i}^{\prime}\right)=h_{N-i+1}^{\prime}, i=1, \cdots, N, \text { for type } A_{2 n-1} \text { or } A_{2 n} ; \\
& \sigma\left(h_{i}^{\prime}\right)=h_{i}^{\prime}, i=1, \cdots, n-1=N-2, \sigma\left(h_{n}^{\prime}\right)=h_{n+1}^{\prime}, \text { for type } D_{n+1} ; \\
& \sigma\left(h_{1}^{\prime}, h_{2}^{\prime}, h_{3}^{\prime}, h_{4}^{\prime}\right)=\left(h_{3}^{\prime}, h_{2}^{\prime}, h_{4}^{\prime}, h_{1}^{\prime}\right) \text { for type } D_{4}^{(3)} .
\end{aligned}
$$

Then the Lie algebra is decomposed as a $\mathbf{Z} / r \mathbf{Z}$-graded Lie algebra:

$$
\mathbf{g}=\mathbf{g}_{0} \oplus \cdots \oplus \mathbf{g}_{r-1},
$$

where $\mathbf{g}_{i}=\left\{x \in \mathbf{g} \mid \sigma(x)=\omega^{i} x\right\}$ and $\omega=e^{2 \pi \sqrt{-1} / r}$. The Dynkin diagram $\Gamma$ decomposes itself into $\sigma$-orbits: $\Gamma=\Gamma_{0} \cup \cdots \cup \Gamma_{r-1}$. It is well-known that the subalgebra $\mathbf{g}_{0}$ is the simple Lie algebra of types $C_{n}, B_{n}, B_{n}$ and $G_{2}$ respectively.

The twisted affine Lie algebras are realized as a subalgebra of the central extension of the loop algebra:

$$
\hat{\mathbf{g}}(\sigma)=\sum_{i} \mathbf{g}_{i \bmod r} \otimes t^{i} \oplus \mathbf{C} c \oplus \mathbf{C} d,
$$

where $c$ is the central element, $d$ is the degree element, and the Lie bracket is given by

$$
\left[x \otimes t^{i}, y \otimes t^{j}\right]=[x y] \otimes t^{i+j}+\frac{i}{r} \delta_{i,-j}\langle x \mid y\rangle c .
$$

The twisted affine Lie algebra $\hat{\mathbf{g}}(\sigma)$ is also generated by the Chevalley generators

$$
e_{i}=E_{i} \otimes t^{\delta_{i 0}}, f_{i}=F_{i} \otimes t^{-\delta_{i 0}}, h_{i}=H_{i} \otimes 1+r a_{\epsilon}^{-1} c, \text { for } i=0,1, \cdots, n,
$$

where $a_{\epsilon}=a_{0}=1$ unless $\mathbf{g}=\mathbf{s} l(2 n+1)$ in which $a_{\epsilon}=a_{n}=2$. (We remark that our choice of $A_{2 n}^{(2)}$ differs from Kac's convention in [14].) The elements $E_{i}, F_{i}, H_{i}$ $(i=0,1, \cdots, n)$ are defined as follows:

$$
\begin{aligned}
& E_{i}=e_{i}^{\prime}, F_{i}=f_{i}, h_{i}=h_{i}^{\prime}, \text { if } \sigma(i)=i ; \\
& E_{i}=\frac{1}{r} \sum_{j=0}^{r-1} e_{\sigma^{j}(i)}^{\prime}, F_{i}=\sum_{j=0}^{r-1} f_{\sigma^{j}(i)}^{\prime}, H_{i}=\frac{1}{r} \sum_{j=0}^{r-1} h_{\sigma^{j}(i)}^{\prime}, \text { if } \sigma(i) \neq i ; \\
& E_{n}=\frac{1}{2}\left(e_{n}^{\prime}+e_{n+1}^{\prime}\right), F_{n}=\left(f_{n}^{\prime}+f_{n+1}^{\prime}\right), H_{n}=\frac{1}{2}\left(h_{n}^{\prime}+h_{n+1}^{\prime}\right) \text { for } A_{2 n}, r=2 ; \\
& E_{0}=\frac{1}{r} \sum_{j=0}^{r-1} \omega^{j} f_{-\sigma^{j}\left(\theta^{0}\right)}^{\prime}, F_{0}=-\sum_{j=0}^{r-1} \omega^{j} e_{\sigma^{j}\left(\theta^{0}\right)}, H_{0}=-\frac{1}{r} \sum_{j=0}^{r-1} h_{-\sigma^{j}\left(\theta^{0}\right)}^{\prime},
\end{aligned}
$$

except for $A_{2 n}, r=2$;

$$
\begin{aligned}
& E_{0}= \frac{1}{r} f_{-\theta^{0}}^{\prime}, F_{0}=-e_{\theta^{0}}^{\prime}, H_{0}=-\frac{1}{r} h_{\theta^{0}}^{\prime}, \text { for } A_{2 n}, r=2 ; \\
& \theta^{0}=\left\{\begin{array}{l}
\alpha_{1}^{\prime}+\cdots+\alpha_{2 n-2}^{\prime}, \text { for } A_{2 n-1}, r=2 \\
\alpha_{1}^{\prime}+\cdots+\alpha_{n}^{\prime}, \text { for } D_{n+1}, r=2 ; \\
\alpha_{1}^{\prime}+\cdots+\alpha_{2 n}^{\prime}, \text { for } A_{2 n}, r=2 \\
\alpha_{1}^{\prime}+\alpha_{2}^{\prime}+\alpha_{3}^{\prime}, \text { for } D_{4}, r=3
\end{array}\right.
\end{aligned}
$$

The above Chevalley generators give a realization of the twisted affine KacMoody algebra $\hat{\mathbf{g}}(A)$ associated to the affine Cartan matrices [14] $A=\left(A_{i j}\right), i, j \in$ 
$I=\{0,1, \cdots, n\}$

$$
\begin{aligned}
& A_{2 n-1}^{(2)}=2 \sum_{i=0}^{n} E_{i i}-\sum_{i=1}^{n-1}\left(E_{i, i+1}+E_{i+1, i}\right)-E_{02}-E_{20}-E_{n-1, n}, \\
& D_{n+1}^{(2)}=2 \sum_{i=0}^{n} E_{i i}-\sum_{i=0}^{n-1}\left(E_{i, i+1}+E_{i+1, i}\right)-E_{01}-E_{n, n-1}, \\
& A_{2 n}^{(2)}=2 \sum_{i=0}^{n} E_{i i}-\sum_{i=0}^{n-1}\left(E_{i, i+1}+E_{i+1, i}\right)-E_{10}-\left(1+\delta_{n, 1}\right) E_{n, n-1}, \\
& D_{4}^{(3)}=2 \sum_{i=0}^{2} E_{i i}-\sum_{i=0}^{2}\left(E_{i, i+1}+E_{i+1, i}\right)-2 E_{12},
\end{aligned}
$$

where the $E_{i j}$ 's are the unit matrices in $\mathbf{Z}^{(n+1) \times(n+1)}$. In the following we will not explicitly list the case $A_{2}^{(2)}$ unless needed. Moreover we define

$$
\alpha_{i}=\frac{1}{p_{i}} \sum_{s=0}^{r-1} \alpha_{\sigma^{s}(i)}^{\prime},
$$

where $p_{i}=1$ if $\sigma(i) \neq i$ and $p_{i}=r$ if $\sigma(i)=i$, and extend it to the weight lattice. It is necessary to normalize the invariant form on $\mathbf{g}_{0}$ by

$$
(\alpha, \beta)=\langle\alpha \mid \beta\rangle / r .
$$

We define the affine root lattice of $\hat{\mathbf{g}}$ to be

$$
\hat{Q}=\mathbf{Z} \alpha_{0} \oplus \cdots \oplus \mathbf{Z} \alpha_{n},
$$

where $\alpha_{i} \in \mathbf{h}^{*}$, and $\left\langle\alpha_{i}, h_{j}\right\rangle=A_{j i},\left\langle\alpha_{i}, d\right\rangle=\delta_{i 0}, i=0,1, \cdots, n$. The affine weight lattice $\hat{P}$ is defined to be

$$
\hat{P}=\mathbf{Z} \Lambda_{0} \oplus \cdots \oplus \mathbf{Z} \Lambda_{n} \oplus \mathbf{Z} \delta,
$$

where $\Lambda_{i}\left(h_{j}\right)=\delta_{i j}, \Lambda_{i}(d)=0$, and $\delta\left(h_{j}\right)=0, \delta(d)=1$ for $j \in I$. The dual affine weight lattice is then defined as

$$
\hat{P}^{\vee}=\mathbf{Z} h_{0} \oplus \mathbf{Z} h_{1} \oplus \cdots \oplus \mathbf{Z} h_{n} \oplus \mathbf{Z} d .
$$

The symmetric bilinear form $\langle\mid\rangle / r$ on $\mathbf{h}^{\prime *}$ induces the normalized symmetric form on $\mathbf{h}^{*}$ denoted by ( | ) (cf. (2.1)) satisfying

$$
\left(\alpha_{i} \mid \alpha_{j}\right)=d_{i} a_{i j}, \quad\left(\delta \mid \alpha_{i}\right)=(\delta \mid \delta)=0 \text { for all } i, j \in I,
$$

where $\left(d_{0}, \cdots, d_{n}\right)=(1, \cdots, 1,2),(1,2, \cdots, 2,1),(2,1, \cdots, 1,1 / 2)$, and $(1,1,3)$, for $A_{2 n-1}^{(2)}, D_{n+1}^{(2)}, A_{2 n}^{(2)}$ and $D_{4}^{(3)}$ respectively.

Let $q_{i}=q^{d_{i}}=q^{\frac{1}{2}\left(\alpha_{i} \mid \alpha_{i}\right)}, i \in I$. The quantum affine algebra $U_{q}\left(X_{N}^{(r)}\right)$ is the associative algebra with 1 over $\mathbf{C}\left(q^{1 / 2}\right)$ generated by the elements $e_{i}, f_{i}(i \in I)$ and 
$q^{h}\left(h \in \hat{P}^{\vee}\right)$ with the following relations:

$$
\begin{aligned}
& q^{h} q^{h^{\prime}}=q^{h+h^{\prime}} \text { for } h, h^{\prime} \in \hat{P}^{\vee}, \quad q^{0}=1, \\
& q^{h} e_{i} q^{-h}=q^{\alpha_{i}(h)} e_{i}, q^{h} f_{i} q^{-h}=q^{-\alpha_{i}(h)} f_{i} \text { for } h \in \hat{P}^{\vee}(i \in I), \\
& e_{i} f_{j}-f_{j} e_{i}=\delta_{i j} \frac{t_{i}-t_{i}^{-1}}{q_{i}-q_{i}^{-1}}, \text { where } t_{i}=q_{i}^{h_{i}}=q^{\frac{1}{2}\left(\alpha_{i} \mid \alpha_{i}\right) h_{i}} \text { and } i, j \in I, \\
& \sum_{m+k=1-a_{i j}}(-1)^{m} e_{i}^{(m)} e_{j} e_{i}^{(n)}=0, \quad \text { and } \\
& \sum_{m+n=1-a_{i j}}(-1)^{m} f_{i}^{(m)} f_{j} f_{i}^{(n)}=0 \text { for } i \neq j,
\end{aligned}
$$

where $e_{i}^{(k)}=e_{i}^{k} /[k]_{i} !, f_{i}^{(k)}=f_{i}^{k} /[k]_{i} !,[m]_{i} !=\prod_{k=1}^{m}[k]_{i}$, and $[k]_{i}=\frac{q_{i}^{k}-q_{i}^{-k}}{q_{i}-q_{i}^{-1}}$. For simplicity we will write $[k]_{i}=[k]$ for $q_{i}=q$. The derived subalgebra generated by $e_{i}, f_{i}, t_{i}(i \in I)$ is denoted by $U_{q}^{\prime}\left(X_{N}^{(r)}\right)$.

The algebra $U_{q}\left(X_{N}^{(r)}\right)$ has a Hopf algebra structure with comultiplication $\Delta$, counit $\varepsilon$, and antipode $S$ defined by

$$
\begin{aligned}
\Delta\left(q^{h}\right) & =q^{h} \otimes q^{h} \text { for } h \in \hat{P}^{\vee}, \\
\Delta\left(e_{i}\right) & =e_{i} \otimes 1+t_{i} \otimes e_{i}, \\
\Delta\left(f_{i}\right) & =f_{i} \otimes t_{i}^{-1}+1 \otimes f_{i} \text { for } i \in I, \\
\varepsilon\left(q^{h}\right) & =1 \text { for } h \in \hat{P}^{\vee}, \\
\varepsilon\left(e_{i}\right) & =\varepsilon\left(f_{i}\right)=0 \text { for } i \in I, \\
S\left(q^{h}\right) & =q^{-h} \text { for } h \in \hat{P}^{\vee}, \\
S\left(e_{i}\right) & =-t_{i}^{-1} e_{i}, \quad S\left(f_{i}\right)=-f_{i} t_{i} \text { for } i \in I .
\end{aligned}
$$

Let $V, W$ be two $U_{q}\left(X_{N}^{(r)}\right)$-modules. The tensor product $V \otimes W$ is defined as the $U_{q}\left(X_{N}^{(r)}\right)$-module via the coproduct $\Delta$. The (restricted) dual $U_{q}\left(X_{N}^{(r)}\right)$-module $V^{*}$ is defined by

$$
\left(x \cdot v^{*}\right)(u)=v^{*}(S(x) \cdot u)
$$

for $x \in U_{q}(\mathbf{g}), u \in V$, and $v^{*} \in V^{*}$.

We now recall Drinfeld's realization of the quantum affine algebra $U_{q}\left(X_{N}^{(r)}\right)$ (and of $\left.U_{q}^{\prime}\left(X_{N}^{(r)}\right)\right)[3,11]$. We will present a slightly different form [11] to avoid the $h$-adic completion.

Let $\omega$ be a primitive $r$ th root. We write $[k]_{j}=\frac{q_{i}^{k}-q_{i}^{-k}}{q_{i}-q_{i}^{-1}}$ and $\alpha_{j}=\alpha_{i}$ if $j$ belongs to the $\sigma$-orbit of $i$, then $[k]_{j}$ or $\alpha_{j}$ is defined for all $j=1, \cdots, N$ though we frequently use only $[k]_{i}$ for $i \in\{0\} \cup \Gamma_{\sigma}=\{0,1, \cdots, n\}$ and $\alpha_{j}$.

Definition 2.1. $[3,11]$ Let $\mathbf{U}$ be the associative algebra with 1 over $\mathbf{C}\left(q^{1 / 2}\right)$ generated by the elements $x_{i}^{ \pm}(k), a_{i}(l), \gamma^{ \pm 1 / 2}, q^{ \pm d}(i=1,2, \cdots, N, k \in \mathbf{Z}, l \in \mathbf{Z} \backslash\{0\})$ 
and $K_{i}^{ \pm 1}\left(i \in \Gamma_{\sigma}=\{1,2, \ldots, n\}\right)$ with the following defining relations:

$$
\begin{aligned}
& x_{\sigma(i)}^{ \pm}(k)=\omega^{k} x_{i}^{ \pm}(k), \quad a_{\sigma(i)}(l)=\omega^{l} a_{i}(l), \\
& {\left[\gamma^{ \pm 1 / 2}, u\right]=0 \text { for all } u \in \mathbf{U},} \\
& {\left[a_{i}(k), a_{j}(l)\right]=\delta_{k+l, 0} \sum_{s=0}^{r-1} \frac{\left[k\left\langle\alpha_{i}^{\prime} \mid \sigma^{s}\left(\alpha_{j}^{\prime}\right)\right\rangle / d_{i}\right]_{i}}{k} \omega^{k s} \frac{\gamma^{k}-\gamma^{-k}}{q_{j}-q_{j}^{-1}},} \\
& {\left[a_{i}(k), K_{j}^{ \pm 1}\right]=\left[q^{ \pm d}, K_{j}^{ \pm 1}\right]=0,} \\
& q^{d} x_{i}^{ \pm}(k) q^{-d}=q^{k} x_{i}^{ \pm}(k), \\
& q^{d} a_{i}(l) q^{-d}=q^{l} a_{i}(l), \\
& K_{i} x_{j}^{ \pm}(k) K_{i}^{-1}=q^{ \pm\left(\alpha_{i} \mid \alpha_{j}\right)} x_{j}^{ \pm}(k), \\
& {\left[a_{i}(k), x_{j}^{ \pm}(l)\right]= \pm \sum_{s=0}^{r-1} \frac{\left[k\left\langle\alpha_{i}^{\prime} \mid \sigma^{s}\left(\alpha_{j}^{\prime}\right)\right\rangle / d_{i}\right]_{i}}{k} \omega^{k s} \gamma^{\mp|k| / 2} x_{j}^{ \pm}(k+l),} \\
& \prod_{s}\left(z-\omega^{s} q^{\left. \pm\left\langle\alpha_{i}^{\prime} \mid \sigma^{s}\left(\alpha_{j}^{\prime}\right)\right\rangle\right)} w\right) x_{i}^{ \pm}(z) x_{j}^{ \pm}(w) \\
& \quad=\prod_{s}\left(z q^{ \pm\left\langle\alpha_{i}^{\prime} \mid \sigma^{s}\left(\alpha_{j}^{\prime}\right)\right\rangle}-\omega^{s} w\right) x_{j}^{ \pm}(w) x_{i}^{ \pm}(z), \\
& {\left[x_{i}^{+}(k), x_{j}^{-}(l)\right]=\sum_{s=0}^{r-1} \frac{\delta_{\sigma^{s}(i), j} \omega^{s l}}{q_{i}-q_{i}^{-1}}\left(\gamma^{\frac{k-l}{2}} \psi_{i}(k+l)-\gamma^{\frac{l-k}{2}} \varphi_{i}(k+l)\right),}
\end{aligned}
$$

where $\psi_{i}(m)$ and $\varphi_{i}(-m)\left(m \in \mathbf{Z}_{\geq 0}\right)$ are defined by

$$
\begin{aligned}
& \sum_{m=0}^{\infty} \psi_{i}(m) z^{-m}=K_{i} \exp \left(\left(q_{i}-q_{i}^{-1}\right) \sum_{k=1}^{\infty} a_{i}(k) z^{-k}\right), \\
& \sum_{m=0}^{\infty} \varphi_{i}(-m) z^{m}=K_{i}^{-1} \exp \left(-\left(q_{i}-q_{i}^{-1}\right) \sum_{k=1}^{\infty} a_{i}(-k) z^{k}\right), \\
& \operatorname{Sym}_{z_{1}, z_{2}} P_{i j}^{ \pm}\left(z_{1}, z_{2}\right) \sum_{s=0}^{2}(-1)^{s}\left[\begin{array}{c}
2 \\
s
\end{array}\right]_{q^{d_{i j}}} x_{i}^{ \pm}\left(z_{1}\right) \cdots x_{i}^{ \pm}\left(z_{s}\right) \\
& x_{j}^{ \pm}(w) x_{i}^{ \pm}\left(z_{s+1}\right) \cdots x_{i}^{ \pm}\left(z_{2}\right)=0, \quad \text { for } A_{i j}=-1, \sigma(i) \neq j, \\
& \left.\operatorname{Sym}_{z_{1}, z_{2}, z_{3}}\left[\left(q^{\mp 3 r / 4} z_{1}-q^{r / 4}+q^{-r / 4}\right) z_{2}+q^{ \pm 3 r / 4} z_{3}\right) x_{i}^{ \pm}\left(z_{1}\right) x_{i}^{ \pm}\left(z_{2}\right) x_{i}^{ \pm}\left(z_{3}\right)\right] \\
& =0, \quad \text { for } A_{i, \sigma(i)}=-1
\end{aligned}
$$

where Sym means the symmetrization over $z_{i}, P_{i j}^{ \pm}(z, w)$ and $d_{i j}$ are defined as follows:

$$
\begin{aligned}
& \text { If } \sigma(i)=i \text {, then } P_{i j}^{ \pm}(z, w)=1 \text { and } d_{i j}=r \text {. } \\
& \text { If } A_{i, \sigma(i)}=0 \text { and } \sigma(j)=j \text {, then } P_{i j}^{ \pm}(z, w)=\frac{z^{r} q^{ \pm 2 r}-w^{r}}{z q^{ \pm 2}-w} \text { and } d_{i j}=r \text {. } \\
& \text { If } A_{i, \sigma(i)}=0 \text { and } \sigma(j) \neq j \text {, then } P_{i j}^{ \pm}(z, w)=1 \text { and } d_{i j}=1 / 2 \text {. } \\
& \text { If } A_{i, \sigma(i)}=-1 \text {, then } P_{i j}^{ \pm}(z, w)=z q^{ \pm r / 2}+w \text { and } d_{i j}=r / 4 \text {. }
\end{aligned}
$$


We denote by $\mathbf{U}^{\prime}$ the subalgebra of $\mathbf{U}$ generated by the elements $x_{i}^{ \pm}(k), a_{i}(l)$, $K_{i}^{ \pm 1}, \gamma^{ \pm 1 / 2}(i=1,2, \cdots, n, k \in \mathbf{Z}, l \in \mathbf{Z} \backslash\{0\})$.

We also need the following explicit isomorphism between the two definitions.

Proposition 2.1. $[3,11]$ The $\mathbf{C}\left(q^{1 / 2}\right)$-algebra isomorphism $\Psi: U_{q}\left(X_{N}^{(r)}\right) \rightarrow \mathbf{U}$ is given by

$$
\begin{aligned}
& e_{i} \mapsto x_{i}^{+}(0), \quad f_{i} \mapsto x_{i}^{-}(0), \quad t_{i} \mapsto K_{i} \quad \text { if } \sigma(i) \neq i, \\
& e_{i} \mapsto x_{i}^{+}(0), \quad f_{i} \mapsto \frac{1}{r} x_{i}^{-}(0), \quad t_{i} \mapsto K_{i} \quad \text { if } \sigma(i)=i, \\
& t_{0} \mapsto \gamma K_{\theta}^{-1}, \quad q^{d} \mapsto q^{d}, \\
& e_{0} \mapsto \frac{1}{2}\left[x_{2}^{-}(0), \cdots\left[x_{n-1}^{-}(0),\left[x_{n}^{-}(0),\left[x_{n-1}^{-}(0), \cdots\right.\right.\right.\right. \\
&\left.\left.\left.\left.\cdots\left[x_{2}^{-}(0), x_{1}^{-}(1)\right]_{q^{-1}} \cdots\right]_{q^{-1}}\right]_{q^{-2}}\right]_{q^{-1}} \cdots\right]_{q^{-1}} K_{\theta}^{-1}, \\
& f_{0} \mapsto-q^{2 n-2} K_{\theta}\left[x_{2}^{+}(0), \cdots\left[x_{n-1}^{+}(0),\left[x_{n}^{+}(0),\left[x_{n-1}^{+}(0), \cdots\right.\right.\right.\right. \\
&\left.\left.\left.\left.\cdots\left[x_{2}^{+}(0), x_{1}^{+}(-1)\right]_{q^{-1}} \cdots\right]_{q^{-1}}\right]_{q^{-2}}\right]_{q^{-1}} \cdots\right]_{q^{-1}}, \text { for } A_{2 n-1}^{(2)} ; \\
&(2.6) e_{0} \mapsto 2^{-n-1}\left[x_{1}^{-}(0), \cdots\left[x_{n-1}^{-}(0), x_{n}^{-}(1)\right]_{q^{-2}} \cdots\right]_{q^{-2}} K_{\theta}^{-1}, \\
& f_{0} \mapsto(-1)^{n-1} q^{2 n-2} K_{\theta}\left[x_{1}^{+}(0), \cdots\left[x_{n-1}^{+}(0), x_{n}^{+}(-1)\right]_{q^{-2}} \cdots\right]_{q^{-2}}, \text { for } D_{n+1}^{(2)} ; \\
& e_{0} \mapsto[2]^{-2 n+2}\left[x_{1}^{-}(0), \cdots\left[x_{n}^{-}(0),\left[x_{n}^{-}(0),\left[x_{n-1}^{-}(0), \cdots\right.\right.\right.\right. \\
&\left.\left.\left.\left.\left.\quad \cdots\left[x_{2}^{-}(0), x_{1}^{-}(1)\right]_{q^{-1}} \cdots\right]_{q^{-1}}\right]_{1}\right]_{q^{-1}} \cdots\right]_{q^{-1}}\right]_{1} K_{\theta}^{-1}, \\
& f_{0} \mapsto(-q)^{2 n-3}[2]^{-2 n+2} K_{\theta}\left[x_{1}^{+}(0), \cdots\left[x_{n}^{+}(0),\left[x_{n}^{+}(0),\left[x_{n-1}^{-}(0), \cdots\right.\right.\right.\right. \\
&\left.\left.\left.\left.\left.\quad \cdots\left[x_{2}^{+}(0), x_{1}^{+}(-1)\right]_{q^{-1}} \cdots\right]_{q^{-1}}\right]_{1}\right]_{q^{-1}} \cdots\right]_{q^{-1}}\right]_{1}, \text { for } A_{2 n}^{(2)} ; \\
& e_{0} \mapsto\left[x_{1}^{-}(0), x_{1}^{-}(0)\right]_{q} K_{\theta}^{-1}, \quad f_{0} \mapsto-q^{-1} K_{\theta}\left[x_{1}^{+}(0), x_{1}^{+}(-1)\right]_{q}, \text { for } A_{2}^{(2)} ; \\
& e_{0} \mapsto \frac{1}{3}\left[x_{1}^{-}(0),\left[x_{2}^{-}(0), x_{1}^{-}(1)\right]_{q^{-3}}\right]_{q^{-1}} K_{\theta}^{-1}, \\
& f_{0} \mapsto q^{4} K_{\theta}\left[x_{1}^{+}(0),\left[x_{2}^{+}(0), x_{1}^{+}(-1)\right]_{q^{-3}}\right]_{q^{-1}}, \text { for } D_{4}^{(3)},
\end{aligned}
$$

where $[x, y]_{v}=x y-v y x$ and $\theta$ is the maximal root in the sense that $\delta=\theta+\alpha_{0}$ (in our convention):

$$
K_{\theta}= \begin{cases}K_{1}\left(K_{2} \cdots K_{n-1}\right)^{2} K_{n}, & \text { for } A_{2 n-1}^{(2)}, \\ K_{1} \cdots K_{n}, & \text { for } D_{n+1}^{(2)}, \\ \left(K_{1} \cdots K_{n}\right)^{2}, & \text { for } A_{2 n}^{(2)}, A_{2}^{(2)}, \\ K_{1} K_{2}^{2}, & \text { for } D_{4}^{(3)} .\end{cases}
$$

The restriction of $\Psi$ to $U_{q}\left(X_{N}^{(r)}\right)$ defines an isomorphism of $U_{q}\left(X_{N}^{(r)}\right)$ and $\mathbf{U}^{\prime}$.

Through the isomorphism of Proposition 2.1 the coproduct will be carried over to the Drinfeld realization.

Theorem 2.2. Let $k \in \mathbf{Z}_{\geq 0}, l \in \mathbf{N}$, and let $N_{+}^{s}$ (resp. $N_{-}^{s}$ ) be the subalgebra of $\mathbf{U}$ generated by the elements $x_{i_{1}}^{+}\left(-m_{1}\right) \cdots x_{i_{s}}^{+}\left(-m_{s}\right)$ (resp. $x_{i_{1}}^{-}\left(m_{1}\right) \cdots x_{i_{s}}^{-}\left(m_{s}\right)$ ) with 
$m_{i} \in \mathbf{Z}_{\geq 0}$. Then the comultiplication $\Delta$ of the algebra $\mathbf{U}$ has the following form:

$$
\begin{aligned}
& \Delta\left(x_{i}^{+}(k)\right)=x_{i}^{+}(k) \otimes \gamma^{k}+\gamma^{2 k} K_{i} \otimes x_{i}^{+}(k) \\
& +\sum_{j=0}^{k-1} \gamma^{\frac{k-j}{2}} \psi_{i}(k-j) \otimes \gamma^{k-j} x_{i}^{+}(j) \quad\left(\bmod N_{-} \otimes N_{+}^{2}\right), \\
& \Delta\left(x_{i}^{+}(-l)\right)=x_{i}^{+}(-l) \otimes \gamma^{-l}+K_{i}^{-1} \otimes x_{i}^{+}(-l) \\
& +\sum_{j=1}^{l-1} \gamma^{\frac{l-j}{2}} \varphi_{i}(-l+j) \otimes \gamma^{-l+j} x_{i}^{+}(-j) \quad\left(\bmod N_{-} \otimes N_{+}^{2}\right), \\
& \Delta\left(x_{i}^{-}(l)\right)=x_{i}^{-}(l) \otimes K_{i}+\gamma^{l} \otimes x_{i}^{-}(l) \\
& +\sum_{j=1}^{l-1} \gamma^{l-j} x_{i}^{-}(j) \otimes \gamma^{\frac{j-l}{2}} \psi_{i}(l-j) \quad\left(\bmod N_{-}^{2} \otimes N_{+}\right), \\
& \Delta\left(x_{i}^{-}(-k)\right)=x_{i}^{-}(-k) \otimes \gamma^{-2 k} K_{i}^{-1}+\gamma^{-k} \otimes x_{i}^{-}(-k) \\
& +\sum_{j=0}^{k-1} \gamma^{j-k} x_{i}^{-}(-j) \otimes \gamma^{\frac{j-k}{2}} \varphi_{i}(j-k) \quad\left(\bmod N_{-}^{2} \otimes N_{+}\right), \\
& \Delta\left(a_{i}(l)\right)=a_{i}(l) \otimes \gamma^{\frac{l}{2}}+\gamma^{\frac{3 l}{2}} \otimes a_{i}(l)\left(\bmod N_{-} \otimes N_{+}\right), \\
& \Delta\left(a_{i}(-l)\right)=a_{i}(-l) \otimes \gamma^{-\frac{3 l}{2}}+\gamma^{-\frac{l}{2}} \otimes a_{i}(-l)\left(\bmod N_{-} \otimes N_{+}\right) .
\end{aligned}
$$

In the above formulas, we only take the nonzero generators: $x_{i}^{ \pm}(k)=a_{i}(k)=0$ if $\sigma(i)=i$ and $k \neq 0 \bmod r$. Moreover the same formulas are true for the derived subalgebra $\mathbf{U}^{\prime}$.

Proof. The proof is an induction on degrees similar to simply-laced types [12]. The formulas of $\Delta\left(x_{i}^{ \pm}(0)\right)$ are clearly valid as given by the isomorphism in Proposition 2.1 and the coproduct (2.4). To get the formulas for degree \pm 1 elements we use the inverse isomorphism $\Psi^{-1}$ : For a sequence $i=i_{1}, i_{2}, \cdots, i_{h^{(r)}-1}$, we have

$$
\begin{aligned}
\Psi^{-1}\left(x_{i}^{+}(-1)\right) & =\text { const } \cdot t_{i_{2}}\left[f_{i_{2}}, t_{i_{3}}\left[f_{i_{3}}, \cdots t_{i_{h-1}}\left[f_{i_{h-1}}, t_{\theta}^{-1} f_{0}\right] \cdots\right]\right] \\
\Psi^{-1}\left(x_{i}^{-}(1)\right) & =\text { const } \cdot t_{i_{2}}^{-1}\left[e_{i_{2}}, t_{i_{3}}^{-1}\left[e_{i_{3}}, \cdots t_{i_{h-1}}^{-1}\left[e_{i_{h-1}}, e_{0} t_{\theta}\right] \cdots\right]\right] .
\end{aligned}
$$

Applying $\Delta$ it follows that

$$
\begin{aligned}
\Delta\left(x_{i}^{+}(-1)\right) & =x_{i}^{+}(-1) \otimes \gamma^{-1}+K_{i}^{-1} \otimes x_{i}^{+}(-1)\left(\bmod N_{-} \otimes N_{+}^{2}\right), \\
\Delta\left(x_{i}^{-}(1)\right) & =x_{i}^{-}(1) \otimes K_{i}+\gamma \otimes x_{i}^{-}(1)\left(\bmod N_{-}^{2} \otimes N_{+}\right) .
\end{aligned}
$$

Using the relations

$$
\begin{aligned}
{\left[x_{i}^{+}(0), x_{i}^{-}(1)\right] } & =p_{i}\left(q_{i}-q_{i}^{-1}\right)^{-1} \gamma^{-1 / 2} \psi_{i}(1)=p_{i} \gamma^{-1 / 2} K_{i} a_{i}(1), \\
{\left[x_{i}^{+}(-1), x_{i}^{-}(0)\right] } & =-p_{i}\left(q_{i}-q_{i}^{-1}\right)^{-1} \gamma^{1 / 2} \varphi_{i}(-1)=p_{i} \gamma^{1 / 2} K_{i}^{-1} a_{i}(-1),
\end{aligned}
$$

where $p_{i}=1$ for $\sigma(i) \neq i$ and $p_{i}=r$ for $\sigma(i)=i$, we obtain

$$
\begin{aligned}
\Delta\left(a_{i}(1)\right) & =a_{i}(1) \otimes \gamma^{1 / 2}+\gamma^{3 / 2} \otimes a_{i}(1)\left(\bmod N_{-} \otimes N_{+}\right), \\
\Delta\left(a_{i}(-1)\right) & =a_{i}(-1) \otimes \gamma^{-3 / 2}+\gamma^{-1 / 2} \otimes a_{i}(-1)\left(\bmod N_{-} \otimes N_{+}\right) .
\end{aligned}
$$

Based on these two formulas and the Drinfeld relations it is easy to see by induction that the formulas of $\Delta\left(x_{i}^{ \pm}(k)\right)$ are valid. Subsequently the formulas for $\Delta\left(a_{i}(k)\right)$ follow from the formulas for $\Delta\left(\phi_{i}(k)\right)$ and $\Delta\left(\psi_{i}(k)\right)$, which proves the theorem. 
We now turn to some special finite dimensional representations $V\left(X_{N}^{(r)}\right)$ of $U_{q}^{\prime}\left(X_{N}^{(r)}\right)$ arising from the level one perfect crystal graphs. We list the representations case by case. Note that in the case of $D_{4}^{(3)}$ the representation graph is different from its crystal one.

For $A_{2 n-1}^{(2)}$,

$$
V=\left(\bigoplus_{i=1}^{n} \mathbf{C}\left(q^{1 / 2}\right) v_{i}\right) \oplus\left(\bigoplus_{i=1}^{n} \mathbf{C}\left(q^{1 / 2}\right) v_{\bar{i}}\right) .
$$

The action is given by

$$
\begin{aligned}
e_{i} & =E_{i, i+1}+E_{\overline{i+1}, \bar{i}}, \quad f_{i}=E_{i+1, i}+E_{\bar{i}, \overline{i+1}}, \\
t_{i} & =q\left(E_{i i}+E_{\overline{i+1}, \overline{i+1}}\right)+q^{-1}\left(E_{\bar{i}, \bar{i}}+E_{i+1, i+1}\right)+\sum_{j \neq i, i+1, \bar{i}, \overline{i+1}} E_{j j}, \\
f_{n} & =E_{\bar{n}, n}, \quad e_{n}=E_{n, \bar{n}}, \\
t_{n} & =q_{n} E_{n n}+q_{n}^{-1} E_{\bar{n}, \bar{n}}+\sum_{j \neq n, \bar{n}} E_{j j}, \\
f_{0} & =E_{2 \overline{1}}+E_{1 \overline{2}}, \quad e_{0}=E_{\overline{1} 2}+E_{\overline{2} 1}, \\
t_{0} & =q\left(E_{\overline{11}}+E_{\overline{22}}\right)+q^{-1}\left(E_{1,1}+E_{2,2}\right)+\sum_{j, \bar{j} \neq 1,2} E_{j j},
\end{aligned}
$$

where $i=1,2, \cdots, n-1,\left(d_{0}, \cdots, d_{n}\right)=(1, \cdots, 1,2)$, and $E_{i j} \in \operatorname{End}(V)$ so that $E_{i j} v_{k}=\delta_{j k} v_{i}$.

For $D_{n+1}^{(2)}$,

$$
V=\left(\bigoplus_{i=0}^{n} \mathbf{C}\left(q^{1 / 2}\right) v_{i}\right) \oplus\left(\bigoplus_{i=0}^{n} \mathbf{C}\left(q^{1 / 2}\right) v_{\bar{i}}\right) .
$$

The action is given by

$$
\begin{aligned}
e_{i} & =E_{i, i+1}+E_{\overline{i+1}, \bar{i}}, \quad f_{i}=E_{i+1, i}+E_{\bar{i}, \overline{i+1}}, \\
t_{i} & =q_{i}\left(E_{i i}+E_{\overline{i+1}, \overline{i+1}}\right)+q_{i}^{-1}\left(E_{\bar{i}, \bar{i}}+E_{i+1, i+1}\right)+\sum_{j \neq i, i+1, \bar{i}, \overline{i+1}} E_{j j}, \\
e_{n} & =[2] E_{n 0}+E_{0 \bar{n}}, \quad f_{n}=[2] E_{\bar{n} 0}+E_{0 n}, \\
t_{n} & =q^{2} E_{n n}+q^{-2} E_{\bar{n}, \bar{n}}+\sum_{j \neq n, \bar{n}} E_{j j}, \\
e_{0} & =[2] E_{\overline{10}}+E_{\overline{0} 1}, \quad f_{0}=[2] E_{1 \overline{0}}+E_{\overline{01}}, \\
t_{0} & =q^{2} E_{\overline{11}}+q^{-2} E_{11}+\sum_{j \neq 1, \overline{1}} E_{j j},
\end{aligned}
$$

where $\left(d_{0}, \cdots, d_{n}\right)=(1,2, \cdots, 2,1)$.

For $A_{2 n}^{(2)}$,

$$
V=\left(\bigoplus_{i=1}^{n} \mathbf{C}\left(q^{1 / 2}\right) v_{i}\right) \oplus \mathbf{C}\left(q^{1 / 2}\right) v_{0} \oplus\left(\bigoplus_{i=1}^{n} \mathbf{C}\left(q^{1 / 2}\right) v_{\bar{i}}\right) .
$$

The action is given by

$$
\begin{aligned}
e_{i} & =E_{i, i+1}+E_{\overline{i+1}, \bar{i}}, \quad f_{i}=E_{i+1, i}+E_{\bar{i}, \overline{i+1}}, \\
t_{i} & =q_{i}\left(E_{i i}+E_{\overline{i+1}, \overline{i+1}}\right)+q_{i}^{-1}\left(E_{\bar{i}, \bar{i}}+E_{i+1, i+1}\right)+\sum_{j \neq i, i+1, \bar{i}, \overline{i+1}} E_{j j}, \\
e_{n} & =[2]_{n} E_{n 0}+E_{0 \bar{n}}, \quad f_{n}=[2]_{n} E_{\bar{n} 0}+E_{0 n}, \\
t_{n} & =q_{n}^{2} E_{n n}+q_{n}^{-2} E_{\bar{n}, \bar{n}}+\sum_{j \neq n, \bar{n}} E_{j j}, \\
e_{0} & =E_{\overline{1} 1}, \quad f_{0}=E_{1 \overline{1}}, \\
t_{0} & =q_{0}\left(E_{\overline{11}}\right)+q_{0}^{-1}\left(E_{11}\right)+\sum_{j \neq 1, \overline{1}} E_{j j},
\end{aligned}
$$

where $\left(d_{0}, d_{1}, \cdots, d_{n}\right)=(2,1, \cdots, 1,1 / 2)$. If $n=1$, then $\left(d_{0}, d_{1}\right)=(4,1)$. 
For $D_{4}^{(3)}$

$$
V=\left(\bigoplus_{i=0}^{3} \mathbf{C}\left(q^{1 / 2}\right) v_{i}\right) \oplus\left(\bigoplus_{i=0}^{3} \mathbf{C}\left(q^{1 / 2}\right) v_{\bar{i}}\right) .
$$

The action is given by

$$
\begin{aligned}
e_{1} & =E_{12}+E_{\overline{2}, \overline{1}}+E_{0 \overline{3}}+[2] E_{30}+E_{3 \overline{0}}, \\
f_{1} & =E_{21}+E_{\overline{1}, \overline{2}}+E_{03}+[2]_{1} E_{\overline{3} 0}+E_{\overline{3}, \overline{0}}, \\
t_{1} & =q\left(E_{11}+E_{\overline{22}}\right)+q^{-1}\left(E_{\overline{11}}+E_{22}\right)+q^{2} E_{33}+q^{-2} E_{\overline{33}}+E_{00}+E_{\overline{00}}, \\
e_{2} & =E_{23}+E_{\overline{32}}, f_{2}=E_{32}+E_{\overline{23}}, \\
t_{2} & =q_{2}\left(E_{22}+E_{\overline{33}}\right)+q_{2}^{-1}\left(E_{33}+E_{\overline{22}}\right)+\sum_{j=0,1, \overline{0}, \overline{1}} E_{j j}, \\
e_{0} & =[2] E_{\overline{10}}+E_{\overline{0} 1}+E_{\overline{3} 2}+E_{\overline{2} 3}+E_{\overline{1}, 0}, \\
f_{0} & =[2] E_{1 \overline{0}}+E_{\overline{01}}+E_{2 \overline{3}}+E_{3 \overline{2}}+E_{10}, \\
t_{0} & =q^{-1}\left(E_{22}+E_{33}\right)+q\left(E_{\overline{22}}+E_{\overline{33}}\right)+q^{-2} E_{11}+q^{2} E_{\overline{11}}+E_{00}+E_{\overline{00}},
\end{aligned}
$$

where $\left(d_{0}, d_{1}, d_{2}\right)=(1,1,3)$. Note that the associated crystal with the last matrices of $e_{1}, f_{1}, e_{0}, f_{0}$ removed is a perfect crystal for $D_{4}^{(3)}$. The perfect crystal structure (see [15]) is more than we will need in what follows.

Since $V$ is a finite dimensional vector space over $\mathbf{C}\left(q^{1 / 2}\right)$, it does not admit a $U_{q}\left(X_{N}^{(r)}\right)$-module structure. However we can define a $U_{q}\left(X_{N}^{(r)}\right)$-module structure on the affinization or evaluation module of $V$. The affinization of $V$ is the $U_{q}\left(X_{N}^{(r)}\right)$ module $V_{z}=V \otimes \mathbf{C}\left(q^{1 / 2}\right)\left[z, z^{-1}\right]$ with the following action:

$$
\begin{aligned}
e_{i}\left(v \otimes z^{m}\right) & =e_{i} v \otimes z^{m+\delta_{i, 0}}, \quad f_{i}\left(v \otimes z^{m}\right)=f_{i} v \otimes z^{m-\delta_{i, 0}}, \\
t_{i}\left(v \otimes z^{m}\right) & =t_{i} v \otimes z^{m} \\
q^{d}\left(v \otimes z^{m}\right) & =q^{m} v \otimes z^{m}
\end{aligned}
$$

for $i=0,1, \cdots, n, m \in \mathbf{Z}, v \in V$.

The evaluation module $V_{z}$ is a level zero $U_{q}\left(X_{N}^{(r)}\right)$-module, i.e., $\gamma$ acts as identity $\left(=q^{0}\right)$. Through the isomorphism $\Psi[11]$ the evaluation module is also a $\mathbf{U}$-module. The action of the Drinfeld generators is given by the following result.

Theorem 2.3. The Drinfeld generators act on the evaluation module $V_{z}$ as follows.

For $V\left(A_{2 n-1}^{(2)}\right)$,

$$
\begin{aligned}
& x_{i}^{+}(k)=\left(q^{i} z\right)^{k} E_{i, i+1}+\left(-q^{2 n-i-1} z\right)^{k} E_{\overline{i+1}, \bar{i}} \\
& x_{i}^{-}(k)=\left(q^{i} z\right)^{k} E_{i+1, i}+\left(-q^{2 n-i-1} z\right)^{k} E_{\bar{i}, \overline{i+1}} \\
& x_{n}^{+}(2 k)=\left(q^{n} z\right)^{2 k} E_{n, \bar{n}} \\
& x_{n}^{-}(2 k)=2\left(q^{n} z\right)^{2 k} E_{\bar{n}, n} \\
& a_{i}(l)=\frac{[l]}{l}\left(\left(q^{i} z\right)^{l}\left(q^{-l} E_{i, i}-q^{l} E_{i+1, i+1}\right)\right. \\
&\left.\quad+\left(-q^{2 n-i} z\right)^{l}\left(q^{-l} E_{\overline{i+1}, \overline{i+1}}-q^{l} E_{\bar{i}, \bar{i}}\right)\right) \\
& a_{n}(2 l)=\frac{[l]_{n}}{l}\left(q^{n} z\right)^{2 l}\left(q^{-2 l} E_{n, n}-q^{2 l} E_{\bar{n}, \bar{n}}\right),
\end{aligned}
$$

where $i=1,2, \cdots, n-1, k \in \mathbf{Z}$, and $l \in \mathbf{Z} \backslash\{0\}, x_{n}^{ \pm}(m)=a_{n}(m)=0$ for $m$ odd, and $q_{n}=q^{2}$. 
For $V\left(D_{n+1}^{(2)}\right)$,

$$
\begin{aligned}
x_{i}^{+}(2 k) & =\left(-q^{2}\right)^{k n} z^{2 k}\left(q^{-2(n-i) k} E_{i, i+1}+q^{2(n-i) k} E_{\overline{i+1}, \bar{i}}\right), \\
x_{i}^{-}(2 k) & =2\left(-q^{2}\right)^{k n} z^{2 k}\left(q^{-2(n-i) k} E_{i+1, i}+q^{2(n-i) k} E_{\bar{i}, \overline{i+1}}\right), \\
a_{i}(2 l) & =\frac{[l] i}{l}\left(-q^{2}\right)^{l n} z^{2 l}\left(q^{-2(n-i+1) l} E_{i, i}-q^{-2(n-i-1) l} E_{i+1, i+1}\right. \\
& \left.+q^{2(n-i-1) l} E_{\overline{i+1}, \overline{i+1}}-q^{2(n-i+1) l} E_{\bar{i}, \bar{i}}\right), \\
x_{n}^{+}(2 k) & =\left(-q^{2}\right)^{k n} z^{2 k}\left([2] E_{n, 0}+E_{0, \bar{n}}\right), \\
x_{n}^{-}(2 k) & =\left(-q^{2}\right)^{k n} z^{2 k}\left(E_{0, n}+[2] E_{\bar{n}, 0}\right), \\
a_{n}(2 l) & =\left(-q^{2}\right)^{n l} z^{2 l} \frac{[2 l]}{2 l}\left(2 q^{-2 l} E_{n, n}\right. \\
x_{n}^{+}(2 k+1) & =\left(-q^{2}\right)^{k n} z^{2 k+1}\left(\left(-q^{2}\right)^{n} E_{\overline{0}, \bar{n}}-[2] E_{n, \overline{0}}\right), \\
x_{n}^{-}(2 k+1) & =\left(-q^{2}\right)^{k n} z^{2 k+1}\left([2] E_{\bar{n}, \overline{0}}-\left(-q^{2}\right)^{n} E_{\overline{0}, n}\right), \\
a_{n}(2 l+1) & =\left(-q^{2}\right)^{n l} z^{2 l+1} \frac{[4 l+2]}{2 l+1}\left(E_{0, \overline{0}}+\left(-q^{2}\right)^{n} E_{\overline{0}, 0}\right),
\end{aligned}
$$

where $i=1,2, \cdots, n-1, k \in \mathbf{Z}$, and $l \in \mathbf{Z} \backslash\{0\}, x_{i}^{ \pm}(m)=a_{i}(m)=0$ for $m$ odd, and $q_{i}=q^{2}$.

For $V\left(A_{2 n}^{(2)}\right)$,

$$
\begin{aligned}
& x_{i}^{+}(k)=\left(q^{i} z\right)^{k}\left(E_{i, i+1}+\left(-q^{2 n-2 i-1} z\right)^{k} E_{\overline{i+1}, \bar{i}}\right), \\
& x_{i}^{-}(k)=\left(q^{i} z\right)^{k}\left(E_{i+1, i}+\left(-q^{2 n-2 i-1} z\right)^{k} E_{\bar{i}, \bar{i}+1}\right), \\
& a_{i}(l)=\frac{[l]}{l}\left(q^{i} z\right)^{l}\left(q^{-l} E_{i, i}-q^{l} E_{i+1, i+1}\right. \\
& \left.+\left(q^{2 n-2 i+1} z\right)^{l}\left(q^{-l} E_{\overline{i+1}, \overline{i+1}}-q^{l} E_{\bar{i}, \bar{i}}\right)\right), \\
& x_{n}^{+}(k)=\left(q^{n} z\right)^{k}\left((-q)^{k} E_{0, \bar{n}}+[2]_{n} E_{n, 0}\right) \text {, } \\
& x_{n}^{-}(k)=\left(q^{n} z\right)^{k}\left(E_{0, n}+(-q)^{k}[2]_{n} E_{\bar{n}, 0}\right) \text {, } \\
& a_{n}(l)=\frac{[2 l]_{n}}{l}\left(q^{n} z\right)^{l}\left(q^{-l} E_{n, n}-q^{l} E_{0,0}+(-q)^{l}\left(q^{-l} E_{0,0}-q^{l} E_{\bar{n}, \bar{n}}\right)\right) \text {, }
\end{aligned}
$$

where $i=1,2, \cdots, n-1, k \in \mathbf{Z}$, and $l \in \mathbf{Z} \backslash\{0\}, q_{0}=q^{2}, q_{i}=q$ and $q_{n}=q^{1 / 2}$.

For $V\left(D_{4}^{(3)}\right)$,

$$
\begin{aligned}
& x_{1}^{+}(3 k)=\left(q^{i} z\right)^{3 k}\left(E_{12}+q^{12 k} E_{\overline{21}}+q^{6 k} E_{0 \overline{3}}+q^{6 k} E_{3 \overline{0}}+q^{6 k}[2] E_{30}\right), \\
& x_{1}^{+}(3 k+1)=\left(q^{i} z\right)^{3 k+1}\left(E_{12}+q^{12 k+4} E_{\overline{21}}+q^{6 k+2}\left(q^{2}-1\right) E_{0 \overline{3}}\right. \\
& \left.-q^{6 k+5} E_{\overline{03}}-q^{6 k}\left(q^{2}+q^{-2}\right) E_{3 \overline{0}}-q^{6 k+3} E_{30}\right), \\
& x_{1}^{+}(3 k+2)=\left(q^{i} z\right)^{3 k}\left(E_{12}+q^{12 k+8} E_{\overline{21}}-q^{6 k+6} E_{0 \overline{3}}\right. \\
& \left.+q^{6 k+7} E_{\overline{03}}+q^{6 k} E_{3 \overline{0}}+q^{6 k+3} E_{30}\right), \\
& x_{1}^{-}(3 k)=\left(q^{i} z\right)^{3 k}\left(E_{21}+q^{12 k} E_{\overline{12}}+q^{6 k} E_{\overline{30}}+q^{6 k}[2] E_{\overline{30}}+q^{6 k} E_{03}\right), \\
& x_{1}^{-}(3 k+1)=\left(q^{i} z\right)^{3 k+1}\left(E_{21}+q^{12 k+4} E_{\overline{12}}+q^{6 k-2} E_{\overline{30}}\right. \\
& \left.-q^{6 k+1} E_{\overline{3} 0}+q^{6 k+5} E_{\overline{0} 3}-q^{6 k+4} E_{03}\right), \\
& x_{1}^{-}(3 k+2)=\left(q^{i} z\right)^{3 k+2}\left(E_{21}+q^{12 k+8} E_{\overline{12}}-q^{6 k+2}\left(q^{2}+q^{-2}\right) E_{\overline{30}}\right. \\
& \left.-q^{6 k+5} E_{\overline{3} 0}-q^{6 k+7} E_{\overline{0} 3}+q^{6 k+4}\left(q^{2}-1\right) E_{03}\right), \\
& x_{2}^{+}(3 k)=\left(q^{2} z\right)^{3 k}\left(E_{23}+q^{6 k} E_{\overline{32}}\right) \text {, } \\
& x_{2}^{-}(3 k)=3(-1)^{k}\left(q^{2} z\right)^{3 k}\left(E_{32}+q^{6 k} E_{\overline{23}}\right) \text {, } \\
& \begin{aligned}
a_{1}(3 l)= & \frac{[3 l]}{3 l}(q z)^{3 l}\left(q^{-3 l} E_{11}-q^{15 l} E_{\overline{11}}-q^{3 l} E_{22}+q^{9 l} E_{\overline{22}}+2 q^{3 l} E_{33}\right. \\
& \left.+\left(q^{3 l}-q^{9 l}\right) E_{00}+\left(q^{3 l}-q^{9 l}\right) E_{\overline{00}}\right),
\end{aligned}
\end{aligned}
$$




$$
\begin{aligned}
& a_{1}(3 k+1)=\frac{[3 k+1]}{3 k+1}(q z)^{3 k+1}\left(q^{-3 k-1} E_{11}-q^{15 k+5} E_{\overline{11}}\right. \\
&-q^{3 k+1} E_{22}+q^{9 k+3} E_{\overline{22}} \\
&+q^{9 k+3} E_{\overline{33}}-q^{3 k+1} E_{33}+\frac{q^{3}+q^{-3}}{q+q^{-1}}\left(q^{3 k+1}+q^{-3 k-1}\right) q^{6 k+1} E_{0 \overline{0}} \\
&-\left(q^{3 k+1}+q^{-3 k-1}\right) q^{6 k+5} E_{\overline{0} 0}+\left(q^{9 k+5}+q^{3 k+3}-q^{3 k+1}\right) E_{00} \\
&\left.+\left(-q^{9 k+5}-q^{3 k+3}+q^{9 k+3}\right) E_{\overline{00}}\right), \\
& a_{1}(3 k+2)=\frac{[3 k+2]}{3 k+2}(q z)^{3 k+2}\left(q^{-3 k-2} E_{11}-q^{15 k+10} E_{\overline{11}}\right. \\
&-q^{3 k+2} E_{22}+q^{9 k+6} E_{\overline{22}} \\
&+q^{9 k+6} E_{\overline{33}}-q^{3 k+2} E_{33}-\frac{q^{3}+q^{-3}}{q+q^{-1}}\left(q^{3 k+2}+q^{-3 k-2}\right) q^{6 k+3} E_{0 \overline{0}} \\
&+\left(q^{3 k+2}+q^{-3 k-2}\right) q^{6 k+7} E_{\overline{00}}+\left(q^{9 k+6}+q^{9 k+8}-q^{3 k+4}\right) E_{00} \\
&\left.+\left(q^{9 k+8}-q^{3 k+4}+q^{3 k+2}\right) E_{\overline{00}}\right), \\
& a_{2}(3 l)=\frac{[l]_{2}}{l}\left(q^{2} z\right)^{3 l}\left(q^{-3 l} E_{22}-q^{3 l} E_{33}+q^{6 l}\left(q^{-3 l} E_{\overline{33}}-q^{3 l} E_{\overline{22}}\right)\right),
\end{aligned}
$$

where $k \in \mathbf{Z}$, and $l \in \mathbf{Z} \backslash\{0\}, q_{0}=q_{1}=q$ and $q_{2}=q^{3}$.

Proof. The theorem is proved by induction using the isomorphism of two definitions. Note that the formulas of degree zero hold by the definitions (2.8), (2.9), (2.10), and (2.11). Now using (2.7) we can get degree one relations, then higher degree relations follow using Drinfeld relations.

Theorem 2.4. The Drinfeld generators act on the dual evaluation module $V_{z}^{*}=$ $V^{*} \otimes \mathbf{C}\left(q^{1 / 2}\right)\left[z, z^{-1}\right]$ as follows.

For $V\left(A_{2 n-1}^{(2)}\right)$,

$$
\begin{aligned}
& x_{i}^{+}(k)=\left(-q^{-1}\right)\left(\left(q^{-i} z\right)^{k} E_{i+1, i}^{*}+\left(-q^{-2 n+i} z\right)^{k} E_{\bar{i}, \bar{i}+1}^{*}\right), \\
& x_{i}^{-}(k)=(-q)\left(\left(q^{-i} z\right)^{k} E_{i, i+1}^{*}+\left(-q^{-2 n+i} z\right)^{k} E_{\bar{i}+1, \bar{i}}^{*}\right) \text {, } \\
& a_{i}(l)=\frac{[l]}{l}\left(\left(q^{-i} z\right)^{l}\left(q^{-l} E_{i+1, i+1}^{*}-q^{l} E_{i i}^{*}\right)\right. \\
& \left.+\left(-q^{-2 n+i} z\right)^{l}\left(q^{-l} E_{\bar{i}, \bar{i}}^{*}-q^{l} E_{\bar{i}+1}^{*}, \overline{i+1}\right)\right), \\
& x_{n}^{+}(2 k)=\left(-q^{-2}\right)\left(\left(q^{-n} z\right)^{2 k} E_{\bar{n}, n}^{*},\right. \\
& x_{n}^{-}(2 k)=2\left(-q^{2}\right)\left(\left(q^{-n} z\right)^{2 k} E_{n, \bar{n}}^{*}\right. \text {, } \\
& a_{n}(2 l)=\frac{[l]_{n}}{l}\left(q^{-n} z\right)^{2 l}\left(\left(q^{-2 l} E_{\bar{n}, \bar{n}}^{*}-q^{2 l} E_{n, n}^{*}\right),\right.
\end{aligned}
$$

where $i=1,2, \cdots, n-1, k \in \mathbf{Z}$, and $l \in \mathbf{Z} \backslash\{0\}$, and $x_{n}^{ \pm}(2 k+1)=a_{n}(2 k+1)=0$.

For $V\left(D_{n+1}^{(2)}\right)$,

$$
\begin{aligned}
x_{i}^{+}(2 k)= & -q^{-2}\left(-q^{2}\right)^{-k n} z^{2 k}\left(q^{2(n-i) k} E_{i+1, i}^{*}+q^{-2(n-i) k} E_{\bar{i}, \overline{i+1}}^{*}\right), \\
x_{i}^{-}(2 k)= & -2 q^{2}\left(-q^{2}\right)^{-k n} z^{2 k}\left(q^{2(n-i) k} E_{i, i+1}^{*}+q^{-2(n-i) k} E_{\overline{i+1}, \bar{i}}^{*}\right), \\
a_{i}(2 l)= & \frac{[l]_{i}}{l}\left(-q^{2}\right)^{-l n} z^{2 l}\left(q^{2(n-i-1) l} E_{i+1, i+1}^{*}-q^{2(n-i+1) l} E_{i, i}^{*}\right. \\
& \left.\quad+q^{-2(n-i+1) l} E_{\bar{i}, \bar{i}}-q^{-2(n-i-1) l} E_{\overline{i+1}, \overline{i+1}}\right), \\
x_{n}^{+}(2 k)= & \left(-q^{2}\right)^{-k n-1} z^{2 k}\left([2] q^{-2} E_{0, n}^{*}+E_{\bar{n}, 0}^{*}\right), \\
x_{n}^{-}(2 k)= & \left(-q^{2}\right)^{-k n+1} z^{2 k}\left(q^{2} E_{n, 0}^{*}+[2] E_{0, \bar{n}}^{*}\right), \\
a_{n}(2 l)= & \left(-q^{2}\right)^{-n l} z^{2 l} \frac{[2 l]}{2 l}\left(2 q^{-2 l} E_{\bar{n}, \bar{n}}^{*}-2 q^{2 l} E_{n, n}^{*}\right. \\
& \quad+\left(q^{-2 l}-q^{2 l}\right)\left(E_{0,0}^{*}-E_{\overline{0}, \overline{0}}^{*}\right),
\end{aligned}
$$




$$
\begin{aligned}
& x_{n}^{+}(2 k+1)=\left(-q^{2}\right)^{-k n-1} z^{2 k+1}\left(\left(-q^{2}\right)^{-n} E_{\bar{n}, \overline{0}}^{*}-[2] q^{-2} E_{\overline{0}, n}^{*}\right), \\
& x_{n}^{-}(2 k+1)=\left(-q^{2}\right)^{-k n+1} z^{2 k+1}\left([2] E_{\overline{0}, \bar{n}}^{*}-q^{2}\left(-q^{2}\right)^{-n} E_{n, \overline{0}}^{*}\right), \\
& a_{n}(2 l+1)=-\left(-q^{2}\right)^{-n l} z^{2 l+1} \frac{[4 l+2]}{2 l+1}\left(E_{\overline{0}, 0}^{*}+\left(-q^{2}\right)^{-n} E_{0, \overline{0}}^{*}\right),
\end{aligned}
$$

where $i=1,2, \cdots, n-1, k \in \mathbf{Z}$, and $l \in \mathbf{Z} \backslash\{0\}, x_{i}^{ \pm}(m)=a_{i}(m)=0$ for $m$ odd, and $q_{i}=q^{2}$.

For $V\left(A_{2 n}^{(2)}\right)$,

$$
\begin{aligned}
x_{i}^{+}(k)= & -q^{-1}\left(q^{-i} z\right)^{k}\left(E_{i+1, i}^{*}+\left(-q^{-2 n+2 i+1} z\right)^{k} E_{\bar{i}, \overline{i+1}}^{*}\right), \\
x_{i}^{-}(k)= & -q\left(q^{-i} z\right)^{k}\left(E_{i, i+1}^{*}+\left(-q^{-2 n+2 i+1} z\right)^{k} E_{\overline{i+1}, \bar{i}}^{*},\right. \\
a_{i}(l)= & \frac{[l]}{l}\left(\left(q^{-i} z\right)^{l}\left(q^{-l} E_{i+1, i+1}^{*}-q^{l} E_{i, i}^{*}\right)\right. \\
& \left.\quad+\left(q^{-2 n+2 i-1} z\right)^{l}\left(q^{-l} E_{\bar{i}, \bar{i}}^{*}-q^{l} E_{\bar{i}+1}^{*}, \overline{i+1}\right)\right), \\
x_{n}^{+}(k)= & -q^{-1}\left(q^{-n} z\right)^{k}\left((-q)^{-k} E_{\bar{n}, 0}^{*}+q^{-1}[2]_{n} E_{0, n}^{*}\right), \\
x_{n}^{-}(k)= & -q\left(q^{-n} z\right)^{k}\left(q E_{n, 0}^{*}+(-q)^{-k}[2]_{n} E_{0, \bar{n}}^{*}\right), \\
a_{n}(l)= & \frac{[2 l]_{n}}{l}\left(q^{-n} z\right)^{l}\left(q^{-l} E_{0,0}^{*}-q^{l} E_{n, n}^{*}\right. \\
& \left.+(-q)^{-l}\left(q^{-l} E_{\bar{n}, \bar{n}}^{*}-q^{l} E_{0,0}\right)\right),
\end{aligned}
$$

where $i=1,2, \cdots, n-1, k \in \mathbf{Z}$, and $l \in \mathbf{Z} \backslash\{0\}, q_{0}=q^{2}, q_{i}=q$ and $q_{n}=q^{1 / 2}$.

For $V\left(D_{4}^{(3)}\right)$,

$$
\begin{aligned}
x_{1}^{+}(3 k)= & \left(-q^{-1}\right)\left(q^{i} z\right)^{3 k}\left(E_{21}^{*}+q^{12 k} E_{\overline{12}}^{*} *\right. \\
& \left.+q^{6 k+1} E_{\overline{3} 0}^{*}+q^{6 k-1} E_{\overline{0} 3}^{*}+q^{6 k-1}[2] E_{03}^{*}\right), \\
x_{1}^{+}(3 k+1)= & \left(-q^{-1}\right)\left(q^{i} z\right)^{3 k+1}\left(E_{21}^{*}+q^{12 k+4} E_{\overline{12}}^{*}\right. \\
& +q^{6 k+2}\left(q^{2}-1\right) E_{\overline{3} 0}^{*}-q^{6 k+6} E_{3 \overline{0}}^{*} \\
& \left.-q^{6 k-1}\left(q^{2}+q^{-2}\right) E_{\overline{0} 3}^{*}-q^{6 k+2} E_{03}^{*}\right), \\
x_{1}^{+}(3 k+2)= & \left(-q^{-1}\right)\left(q^{i} z\right)^{3 k}\left(E_{21}^{*}+q^{12 k+8} E_{\overline{12}}^{*}-q^{6 k+7} E_{\overline{3} 0}^{*}+q^{6 k+8} E_{\overline{30}}^{*}\right. \\
& \left.+q^{6 k-1} E_{\overline{0} 3}^{*}+q^{6 k+2} E_{03}^{*}\right), \\
x_{1}^{-}(3 k)= & (-q)\left(q^{i} z\right)^{3 k}\left(E_{12}^{*}+q^{12 k} E_{\overline{21}}^{*}\right. \\
& \left.+q^{6 k-1} E_{03}^{*}+q^{6 k-1}[2] E_{0 \overline{3}}^{*}+q^{6 k+1} E_{30}^{*}\right), \\
x_{1}^{-}(3 k+1)= & (-q)\left(q^{i} z\right)^{3 k+1}\left(E_{12}^{*}+q^{12 k+4} E_{\overline{21}}^{*}+q^{6 k-3} E_{\overline{03}}^{*}\right. \\
& \left.-q^{6 k} E_{0 \overline{3}}^{*}+q^{6 k+6} E_{3 \overline{0}}^{*}-q^{6 k+5} E_{30}^{*}\right), \\
x_{1}^{-}(3 k+2)= & (-q)\left(q^{i} z\right)^{3 k+2}\left(E_{12}^{*}+q^{12 k+8} E_{\overline{21}}^{*}\right. \\
& -q^{6 k+1}\left(q^{2}+q^{-2}\right) E_{\overline{03}}^{*}-q^{6 k+4} E_{0 \overline{3}}^{*} \\
& \left.-q^{6 k+8} E_{30}^{*}+q^{6 k+5}\left(q^{2}-1\right) E_{30}^{*}\right), \\
x_{2}^{+}(3 k)= & \left(-q^{-3}\right)\left(q^{2} z\right)^{3 k}\left(E_{32}^{*}+q^{6 k} E_{\overline{23}}^{*}\right), \\
x_{2}^{-}(3 k)= & 3\left(-q^{3}\right)(-1)^{k}\left(q^{2} z\right)^{3 k}\left(E_{23}^{*}+q^{6 k} E_{\overline{32}}^{*}\right),
\end{aligned}
$$




$$
\begin{aligned}
a_{1}(3 l)= & \frac{[3 l]}{3 l}\left(q^{-1} z\right)^{3 l}\left(-q^{3 l} E_{11}^{*}+q^{-15 l} E_{\overline{11}}^{*}+q^{-3 l} E_{22}^{*}\right. \\
& -q^{-9 l} E_{\overline{22}}^{*}-2 q^{-3 l} E_{33}^{*} \\
& \left.-\left(q^{-3 l}-q^{-9 l}\right) E_{00}^{*}-\left(q^{-3 l}-q^{-9 l}\right) E_{\overline{00}}^{*}\right), \\
a_{1}(3 k+1)= & \frac{[3 k+1]}{3 k+1}\left(q^{-1} z\right)^{3 k+1}\left(-q^{3 k+1} E_{11}^{*}+q^{-15 k-5} E_{\overline{11}}^{*}+q^{-3 k-1} E_{22}^{*}\right. \\
& -q^{-9 k-3} E_{\overline{22}}^{*}-q^{-9 k-3} E_{\overline{33}}^{*}-q^{-3 k-1} E_{33}^{*} \\
& -\frac{q^{3}+q^{-3}}{q+q^{-1}}\left(q^{-3 k-1}+q^{3 k+1}\right) q^{-6 k-1} E_{\overline{0} 0}^{*} \\
& +\left(q^{-3 k-1}+q^{3 k+1}\right) q^{-6 k-5} E_{0 \overline{0}}^{*} \\
& -\left(q^{-9 k-5}+q^{-3 k-3}-q^{-3 k-1}\right) E_{00}^{*} \\
& \left.-\left(-q^{-9 k-5}-q^{-3 k-3}+q^{-9 k-3}\right) E_{\overline{00}}^{*}\right), \\
a_{1}(3 k+2)= & \frac{[3 k+2]}{3 k+2}\left(q^{-1} z\right)^{3 k+2}\left(-q^{3 k+2} E_{11}^{*}+q^{-15 k-10} E_{\overline{11}}^{*}\right. \\
& +q^{-3 k-2} E_{22}^{*}-q^{-9 k-6} E_{\overline{22}}^{*}-q^{-9 k-6} E_{\overline{33}}^{*} \\
& +q^{-3 k-2} E_{33}^{*}+\frac{q^{3}+q^{-3}}{q+q^{-1}}\left(q^{-3 k-2}+q^{3 k+2}\right) q^{-6 k-3} E_{\overline{0} 0}^{*} \\
& -\left(q^{-3 k-2}+q^{3 k+2}\right) q^{-6 k-7} E_{0 \overline{0}}^{*} \\
& -\left(q^{-9 k-6}+q^{-9 k-8}-q^{-3 k-4}\right) E_{00}^{*} \\
& \left.-\left(q^{-9 k-8}-q^{-3 k-4}+q^{-3 k-2}\right) E_{\overline{00}}^{*}\right), \\
a_{2}(3 l)= & \frac{[l]_{2}}{l}\left(q^{-2} z\right)^{3 l}\left(-q^{3 l} E_{22}^{*}+q^{-3 l} E_{33}^{*}-q^{-6 l}\left(q^{3 l} E_{\overline{33}}^{*}+q^{-3 l} E_{\overline{22}}^{*}\right)\right) . \\
& -
\end{aligned}
$$

Proof. Recall that the dual module $W^{*}$ of a module $W$ is defined by

$$
a \cdot u^{*}(v)=u^{*}(S(a) \cdot v)
$$

for $a \in U_{q}\left(X_{N}^{(r)}\right), u^{*} \in W^{*}$ and $v \in W$. Explicitly we have the following description of the dual modules. We only write down the matrices for the generators. The underlying space is the standard dual vector space $V^{*}$ spanned by $v_{i}^{*}, i$ runs through the index set of various space $V$. We also use $E_{i j}^{*} \in \operatorname{End}\left(V^{*}\right)$ so that $E_{i j}^{*} v_{k}^{*}=\delta_{j k} v_{i}^{*}$.

For $A_{2 n-1}^{(2)}$,

$$
V=\left(\bigoplus_{i=1}^{n} \mathbf{C}\left(q^{1 / 2}\right) v_{i}\right) \oplus\left(\bigoplus_{i=1}^{n} \mathbf{C}\left(q^{1 / 2}\right) v_{\bar{i}}\right)
$$

The action is given by

$$
\begin{aligned}
& e_{i}=-q^{-1}\left(E_{i+1, i}^{*}+E_{\bar{i}, \bar{i}+1}^{*}\right), \quad f_{i}=-q\left(E_{i, i+1}^{*}+E_{\bar{i}+1, \bar{i}}^{*}\right), \\
& t_{i}=q^{-1}\left(E_{i i}^{*}+E_{\overline{i+1}, \bar{i}+1}^{*}\right)+q\left(E_{\bar{i}, \bar{i}}^{*}+E_{i+1, i+1}^{*}\right)+\sum_{j \neq i, i+1, \bar{i}, \bar{i}+1} E_{j j}^{*}, \\
& f_{n}=-q_{n}^{-1} E_{n, \bar{n}}^{*}, \quad e_{n}=-q_{n} E_{\bar{n}, n}^{*},
\end{aligned}
$$




$$
\begin{aligned}
t_{n} & =q_{n}^{-1} E_{n n}^{*}+q_{n} E_{\bar{n}, \bar{n}}^{*}+\sum_{j \neq n, \bar{n}} E_{j j}^{*}, \\
e_{0} & =-q^{-1}\left(E_{2 \overline{1}}^{*}+E_{1 \overline{2}}^{*}\right), \quad f_{0}=-q\left(E_{\overline{1} 2}^{*}+E_{\overline{2} 1}^{*}\right), \\
t_{0} & =q^{-1}\left(E_{\overline{11}}^{*}+E_{\overline{2}, \overline{2}}^{*}\right)+q\left(E_{1,1}^{*}+E_{2,2}^{*}\right)+\sum_{j, \bar{j} \neq 1,2} E_{j j}^{*},
\end{aligned}
$$

where $i=1,2, \cdots, n-1,\left(d_{0}, \cdots, d_{n}\right)=(1, \cdots, 1,2)$.

For $D_{n+1}^{(2)}$,

$$
\begin{aligned}
e_{i} & =-q^{-2}\left(E_{i+1, i}^{*}+E_{\bar{i}, \overline{i+1}}^{*}\right), \quad f_{i}=-q^{2}\left(E_{i, i+1}^{*}+E_{\overline{i+1}, \bar{i}}^{*}\right), \\
t_{i} & =q^{-2}\left(E_{i i}^{*}+E_{\bar{i}+1, \bar{i}+1}^{*}\right)+q^{2}\left(E_{\bar{i}, \bar{i}}^{*}+E_{i+1, i+1}^{*}\right)+\sum_{j \neq i, i+1, \bar{i}, \overline{i+1}} E_{j j}^{*}, \\
e_{n} & =-q^{-2}\left([2] E_{0, n}^{*}+q^{2} E_{\bar{n}, 0}^{*}\right), \quad f_{n}=-q^{2}\left(q^{-2}[2] E_{0, \bar{n}}^{*}+E_{n, 0}^{*}\right), \\
t_{n} & =q^{-2} E_{n n}^{*}+q^{2} E_{\bar{n}, \bar{n}}^{*}+\sum_{j \neq n, \bar{n}} E_{j j}^{*}, \\
e_{0} & =-\left([2] q^{-2} E_{\overline{01}}^{*}+E_{1, \overline{0}}^{*}\right), f_{0}=-\left([2] E_{\overline{0}, 1}^{*}+q^{2} E_{\overline{10}}^{*}\right), \\
t_{0} & =q^{-2} E_{\overline{11}}^{*}+q^{2}\left(E_{11}^{*}+\sum_{j \neq 1, \overline{1}} E_{j j}^{*}\right),
\end{aligned}
$$

where $\left(d_{0}, \cdots, d_{n}\right)=(1,2, \cdots, 2,1)$.

For $A_{2 n}^{(2)}$,

$$
\begin{aligned}
e_{i} & =-q^{-1}\left(E_{i+1, i}^{*}+E_{\bar{i}, \overline{i+1}}^{*}\right), \quad f_{i}=-q\left(E_{i, i+1}^{*}+E_{\overline{i+1}, \bar{i}}^{*},\right. \\
t_{i} & =q^{-1}\left(E_{i i}^{*}+E_{\overline{\bar{i}}}^{*}, \overline{i+1}\right)+q\left(E_{\bar{i}, \bar{i}}^{*}+E_{i+1, i+1}^{*}\right)+\sum_{j \neq i, i+1, \bar{i}, \overline{i+1}} E_{j j}^{*}, \\
e_{n} & =-q^{-1}\left([2]_{n} E_{0, n}^{*}+q E_{\bar{n}, 0}^{*}\right), \quad f_{n}=-q\left(q^{-1}[2]_{n} E_{0, \bar{n}}^{*}+E_{n, 0}^{*}\right), \\
t_{n} & =q^{-1} E_{n n}^{*}+q E_{\bar{n}, \bar{n}}^{*}+\sum_{j \neq n, \bar{n}} E_{j j}^{*}, \\
e_{0} & =-q^{-2} E_{1, \overline{1}}^{*}, \quad f_{0}=-q^{2} E_{\overline{1}, 1}^{*}, \\
t_{0} & =q_{0}^{-1} E_{\overline{11}}^{*}+q_{0} E_{11}^{*}+\sum_{j \neq 1, \overline{1}} E_{j j}^{*},
\end{aligned}
$$

where $\left(d_{0}, d_{1}, \cdots, d_{n}\right)=(2,1, \cdots, 1,1 / 2)$.

For $D_{4}^{(3)}$,

$$
\begin{aligned}
e_{1} & =\left(-q^{-1}\right)\left(E_{21}^{*}+E_{\overline{1}, \overline{2}}^{*}+q E_{\overline{30}}^{*}+q^{-1}[2] E_{03}^{*}+q^{-1} E_{\overline{03}}^{*}\right), \\
f_{1} & =-q\left(E_{12}^{*}+E_{\overline{2}, \overline{1}}^{*}+q E_{30}^{*}+q^{-1}[2]_{1} E_{0 \overline{3}}^{*}+q^{-1} E_{\overline{03}}^{*},\right. \\
t_{1} & =q^{-1}\left(E_{11}^{*}+E_{\overline{22}}^{*}\right)+q\left(E_{\overline{11}}^{*}+E_{22}^{*}\right)+q^{-2} E_{33}^{*}+q^{2} E_{\overline{33}}^{*}+E_{00}^{*}+E_{\overline{00}}^{*}, \\
e_{2} & =\left(-q^{-3}\right)\left(E_{32}^{*}+E_{\overline{23}}^{*}\right), f_{2}=\left(-q^{3}\right)\left(E_{23}^{*}+E_{\overline{32}}^{*}\right), \\
t_{2} & =q^{-3}\left(E_{22}^{*}+E_{\overline{33}}^{*}\right)+q^{3}\left(E_{33}^{*}+E_{\overline{22}}^{*}\right)+\sum_{j=0,1, \overline{0}, \overline{1}} E_{j j}^{*}, \\
e_{0} & =\left(-q^{-1} z\right)\left(q^{-1}[2] E_{01}^{*}+q E_{1 \overline{0}}^{*}+E_{2 \overline{3}}^{*}+E_{33}^{*}+q^{-1} E_{01}^{*}\right), \\
f_{0} & =\left(-q z^{-1}\right)\left(q^{-1}[2] E_{\overline{0} 1}^{*}+q E_{\overline{10}}^{*}+E_{\overline{3} 2}^{*}+E_{\overline{2} 3}^{*}+q^{-1} E_{01}^{*}\right), \\
t_{0} & =q\left(E_{22}^{*}+E_{33}^{*}\right)+q^{-1}\left(E_{\overline{22}}^{*}+E_{\overline{33}}^{*}+q^{2} E_{11}^{*}+q^{-2} E_{\overline{11}}^{*}+E_{00}^{*}+E_{\overline{00}}^{*},\right.
\end{aligned}
$$

where $\left(d_{0}, d_{1}, d_{2}\right)=(1,1,3)$.

In the above formulas, $i=1,2, \cdots, n-1$. They show that the Drinfeld generators of degree zero elements do act on $V^{*}$ as given in the theorem. The higher degree generators are obtained by inductions using Drinfeld relations by the same argument as in Theorem 2.3.

\section{LeVel one Realizations of $U_{q}\left(X_{N}^{(r)}\right)$}

In this section we recall the construction of level one twisted quantum affine algebras given in [9]. The level one integrable modules are $V\left(\Lambda_{0}\right), V\left(\Lambda_{1}\right)$ for $A_{2 n-1}^{(2)}$; $V\left(\Lambda_{0}\right), V\left(\Lambda_{n}\right)$ for $D_{n+1}^{(2)} ; V\left(\Lambda_{n}\right)$ for $A_{2 n}^{(2)}$; and $V\left(\Lambda_{0}\right)$ for $D_{4}^{(3)}$.

Let $Q^{\prime}$ be the root lattice of the simply-laced finite dimensional Lie algebra of types $A_{2 n-1}, D_{n+1}, A_{2 n}, D_{4}$. We shall use the same convention or notation as in 
section 1. The diagram automorphism $\sigma$ decomposes $Q^{\prime}=\mathbf{Z} \alpha_{1}^{\prime} \oplus \cdots \oplus \mathbf{Z} \alpha_{N}^{\prime}$ into orbits: $Q^{\prime}=Q_{0}^{\prime} \oplus Q_{1}^{\prime} \cdots \oplus Q_{r-1}^{\prime}$. We shall also use $Q$ to denote the fixed-point sublattice $Q_{0}^{\prime}$. We extend the action of $\sigma$ to the weight lattice $P^{\prime}=\mathbf{Z} \lambda_{1}^{\prime} \oplus \cdots \oplus \mathbf{Z} \lambda_{N}^{\prime}$; accordingly, we have $P^{\prime}=P_{0}^{\prime} \oplus \cdots \oplus P_{r-1}^{\prime}$. Likewise we identify $P$ with $P_{0}^{\prime}$. Clearly $P$ and $Q$ are the weight and root lattices of the root system of types $C_{n}, B_{n}, 2 B_{n}$ and $G_{2}$ respectively.

Using the same notation as in section 1, the virtual finite dimensional fundamental weights are given by

$$
\begin{aligned}
\lambda_{1}= & \\
\lambda_{n}=2 \alpha_{1}+\cdots+2 \alpha_{n-1}+\alpha_{n}, & \text { for } A_{2 n-1}^{(2)}, \\
\left(\lambda_{1}^{\prime}+\lambda_{2}^{\prime}\right)=\left(\alpha_{1}^{\prime}+\cdots+\alpha_{2 n}^{\prime}\right) & \\
=2 \alpha_{1}+\cdots+2 \alpha_{n}, & \text { for } A_{2 n}^{(2)}, \\
\lambda_{1}^{\prime}=\alpha_{1}^{\prime}+\cdots+\alpha_{n-1}^{\prime}+\frac{1}{2}\left(\alpha_{n}^{\prime}+\alpha_{n+1}^{\prime}\right) & \\
=\alpha_{1}+\cdots+\alpha_{n}, & \text { for } D_{n+1}^{(2)}, \\
\left(\lambda_{1}^{\prime}+\lambda_{2}^{\prime}+\lambda_{3}^{\prime}\right)=2\left(\alpha_{1}^{\prime}+\alpha_{3}^{\prime}+\alpha_{4}^{\prime}\right)+3 \alpha_{2}^{\prime} & \\
=2 \alpha_{1}+3 \alpha_{2}, & \text { for } D_{4}^{(3)}, \\
\lambda_{n}+\lambda_{n+1}^{\prime}=\alpha_{1}^{\prime}+\cdots+(n-1) \alpha_{n-1}^{\prime}+\frac{n}{2}\left(\alpha_{n}^{\prime}+\alpha_{n+1}^{\prime}\right) & \alpha_{1}+2 \alpha_{2}+\cdots+(n-1) \alpha_{n}+\frac{n}{2} \alpha_{n}, \text { for } D_{n+1}^{(2)} \cdot
\end{aligned}
$$

Note that $\Lambda_{0}$ or $\Lambda_{n}$ (for $A_{2 n}^{(2)}$ ) is the basic level one weight.

Let $\omega_{0}=(-1)^{r} \omega$. There exists a unique central extension

$$
1 \longrightarrow\left\langle\omega_{0}\right\rangle \longrightarrow \hat{Q}^{\prime} \stackrel{-}{\longrightarrow} Q^{\prime} \longrightarrow 1
$$

of $Q^{\prime}$ by the cyclic group $\left\langle\omega_{0}\right\rangle$ with the commutator map $C$ defined below:

$$
\begin{aligned}
a b a^{-1} b^{-1} & =C(\bar{a}, \bar{b}) \quad \text { for } a, b \in \hat{Q}^{\prime} \\
& =\prod_{s \in \mathbf{Z} / r \mathbf{Z}}\left(-\omega^{s}\right)^{\left\langle\bar{a} \mid \sigma^{s} \bar{b}\right\rangle}
\end{aligned}
$$

We remark that the commutator map $C$ has the following properties:

$$
\begin{aligned}
& C\left(\alpha^{\prime}+\beta^{\prime}, \gamma^{\prime}\right)=C\left(\alpha^{\prime}, \gamma^{\prime}\right) C\left(\beta^{\prime}, \gamma^{\prime}\right) \\
& C\left(\alpha^{\prime}, \beta^{\prime}+\gamma^{\prime}\right)=C\left(\alpha^{\prime}, \beta^{\prime}\right) C\left(\alpha^{\prime}, \gamma^{\prime}\right) \\
& C\left(\alpha^{\prime}, \alpha^{\prime}\right)=1 \\
& C\left(\sigma \alpha^{\prime}, \sigma \beta^{\prime}\right)=C\left(\alpha^{\prime}, \beta^{\prime}\right)
\end{aligned}
$$

for $\alpha^{\prime}, \beta^{\prime}, \gamma^{\prime} \in Q^{\prime}$. Also $C\left(\alpha^{\prime}, \beta^{\prime}\right)=C\left(\beta^{\prime}, \alpha^{\prime}\right)^{-1}$.

The automorphism $\sigma$ can be lifted to an automorphism $\hat{\sigma}$ of the extension $\hat{Q}^{\prime}$ of $Q^{\prime}$ such that

$$
\begin{aligned}
(\hat{\sigma} a)^{-} & =\sigma \bar{a}, & & \text { for all } a \in \hat{Q}^{\prime} \\
\hat{\sigma} a & =a & & \text { whenever } \sigma \bar{a}=\bar{a} .
\end{aligned}
$$

Let $\alpha^{\prime} \rightarrow e_{\alpha^{\prime}}$ be a section of $\hat{Q}^{\prime}$ such that $e_{0}=1$. Thus $e_{\alpha_{i}^{\prime}}, i=1, \ldots, \ell$, are generators of $\mathbf{C}[\hat{Q}]$.

We consider the complementary subspace

$$
Q^{\perp}=\left\{\alpha \in Q^{\prime} \mid\left\langle\alpha, \alpha_{i}\right\rangle=0, i \in \Gamma_{0}\right\}=(1-\sigma) Q^{\prime}
$$


using the diagram automorphism $\sigma$. By $\hat{Q}^{\perp}$ we mean the pullback of $Q^{\perp}$ to $\hat{Q}^{\prime}$. Notice that on $Q^{\perp}$ we have $C_{Q^{\perp}}(\alpha, \beta)=\omega^{\left\langle\sum s \sigma^{s} \alpha, \beta\right\rangle}$. Using (3.1) we see that there exists a unique character $\tau: \hat{Q}^{\perp} \longrightarrow \mathbf{C}^{\times}$such that

$$
\tau\left(\omega_{0}\right)=\omega_{0}, \quad \tau\left(a \hat{\sigma} a^{-1}\right)=\omega^{-\left\langle\sum \sigma^{s} \bar{a} \mid \bar{a}\right\rangle / 2}, \text { for } a \in \hat{Q}^{\prime} .
$$

We form the induced $\hat{Q}^{\prime}$-module

$$
\mathbf{C}\{Q\}=\mathbf{C}\left[\hat{Q}^{\prime}\right] \otimes_{\mathbf{C}_{\left[\hat{Q}^{\perp}\right]}} \mathbf{C}_{\tau}
$$

where $\mathbf{C}_{\tau}$ is the 1-dimensional $\hat{Q}^{\perp}$-module afforded by the character $\tau$. The use of the notation is justified by the vector space isomorphism $V \simeq \mathbf{C}\left[Q^{\prime} / Q^{\tau}\right]$ induced from the natural projection from $Q^{\prime}$ to $Q_{0}^{\prime}=Q$.

On $\mathbf{C}\{Q\}$ the action of $\hat{Q}^{\prime}$ and $Q=\left\{\alpha \in Q^{\prime} \mid \sigma(\alpha)=\alpha\right\}$ are as follows:

$$
\begin{array}{ccc}
a \cdot b \otimes t & =a b \otimes t, & a, b \in \hat{Q}^{\prime}, t \in T, \\
\alpha \cdot b \otimes t & =\langle\alpha \mid \bar{b}\rangle b \otimes t, & \alpha \in Q .
\end{array}
$$

We define $z^{\alpha(0)}\left(\alpha \in Q^{\prime}\right)$ as an operator on $V$ via

$$
z^{\alpha(0)} \cdot b \otimes t=z^{\langle\alpha, \bar{b}\rangle} b \otimes t
$$

and the operator $\omega^{\alpha(0)}$ on $U$ by $\omega^{\alpha(0)} \cdot b \otimes t=\omega^{\langle\alpha, \bar{b}\rangle} b \otimes t$.

We have as operators on $V$

$$
\begin{aligned}
z^{\alpha(0)} a & =a z^{\alpha(0)+\langle\alpha, \bar{a}\rangle}, \\
\omega^{\alpha(0)} a & =a \omega^{\alpha(0)+\langle\alpha, \bar{a}\rangle}, \\
\hat{\sigma} a & =a \omega^{\Sigma \sigma^{r} \bar{a}(0)+\left\langle\bar{a}, \Sigma \sigma^{r} \bar{a}\right\rangle / 2} .
\end{aligned}
$$

For $\alpha=\sum m_{i} \alpha_{i}^{\prime} \neq 0$, we define

$$
e_{\alpha}=e_{\alpha_{1}^{\prime}}^{m_{1}} \cdots e_{\alpha_{N}^{\prime}}^{m_{N}}
$$

where the order is fixed. We then have

$$
e_{\alpha_{i}^{\prime}} e_{-\alpha_{i}^{\prime}}=\omega^{\sum\left\langle\alpha_{i}^{\prime} \mid \sigma^{s}\left(\alpha_{i}^{\prime}\right)\right\rangle}, \quad e^{\alpha_{i}^{\prime}} e^{\alpha_{j}^{\prime}}=(-\omega)^{\left\langle\alpha_{i}^{\prime} \mid \alpha_{j}^{\prime}\right\rangle} e^{\alpha_{j}^{\prime}} e^{\alpha_{i}^{\prime}} .
$$

Subsequently we have

$$
e_{\alpha} e_{\beta}=\omega^{\sum_{s=0}^{r-1}\left\langle\alpha \mid \sigma^{s}(\beta)\right\rangle} e_{\beta} e_{\alpha}
$$

for any $\alpha, \beta \in Q^{\prime}$.

Later we want to use freely $Q$, as fixed point sublattice of $Q^{\prime}$, thus we are doing this not only for $Q^{\prime}$ but also for $Q$. For $\lambda \in P^{\prime}$ we define the vector space $\mathbf{C}\{Q\} e_{\lambda}$ as a $\mathbf{C}\{Q\}$-module by formally adjoining the element $e_{\lambda}$. Then we can define the operator $e^{\lambda}: \mathbf{C}\{Q\} \mapsto \mathbf{C}\{Q\} e_{\lambda}$ naturally.

For $\alpha \in P$ define the operator $\partial_{\alpha}$ on $\mathbf{C}\{Q\}$ or $\mathbf{C}\{Q\} e^{\lambda}$ by

$$
\partial_{\alpha} e^{\beta}=(\alpha \mid \beta) e^{\beta} .
$$

Let $U_{q}(\hat{\mathbf{h}})$ be the infinite dimensional Heisenberg algebra generated by $a_{i}(k)$ and the central element $\gamma(k \in \mathbf{Z} \backslash\{0\}, i=1, \cdots, n)$ subject to the relations

$$
\begin{gathered}
a_{\sigma(i)}(k)=a_{i}(k) \omega^{k}, \\
{\left[a_{i}(k), a_{j}(l)\right]=\delta_{k+l, 0} \sum_{s=0}^{r-1} \frac{\left[\left\langle\alpha_{i}^{\prime} \mid \sigma^{s}\left(\alpha_{j}^{\prime}\right)\right\rangle k / d_{i}\right]_{i}}{k} \omega^{s k} \frac{\gamma^{k}-\gamma^{-k}}{q_{j}-q_{j}^{-1}} .}
\end{gathered}
$$


The algebra $U_{q}(\hat{\mathbf{h}})$ acts on the space of symmetric algebra $\operatorname{Sym}\left(\hat{\mathbf{h}}_{\sigma}^{-}\right)$generated by $a_{i}(-k)(k \in \mathbf{N}, i=1, \cdots, n)$ with $\gamma=q$ by the action

$$
\begin{aligned}
a_{i}(-k) & \mapsto \text { multiplication by } a_{i}(-k), \\
a_{i}(k) & \mapsto \sum_{s=0}^{r-1} \sum_{j} \frac{\left[\left\langle\alpha_{i}^{\prime} \mid \sigma^{s}\left(\alpha_{j}^{\prime}\right)\right\rangle k / d_{i}\right]_{i}}{k} \omega^{s k} \frac{q^{k}-q^{-k}}{q_{j}-q_{j}^{-1}} \frac{d}{d a_{j}(-k)} .
\end{aligned}
$$

For convenience we also identify $\operatorname{Sym}\left(\hat{\mathbf{h}}_{\sigma}^{-}\right)$with $\operatorname{Sym}\left(\hat{\mathbf{h}}^{-}\right)$by modulo the relations (3.6).

We define

$$
\begin{aligned}
W_{\epsilon} & =\operatorname{Sym}\left(\hat{\mathbf{h}}_{\sigma}^{-}\right) \otimes \mathbf{C}\{Q\}, \text { for all cases of basic modules, } \\
W_{1} & =\operatorname{Sym}\left(\hat{\mathbf{h}}_{\sigma}^{-}\right) \otimes \mathbf{C}\{Q\} e_{\lambda_{1}}, \text { for } A_{2 n-1}^{(2)} \\
W_{n} & =\operatorname{Sym}\left(\hat{\mathbf{h}}_{\sigma}^{-}\right) \otimes \mathbf{C}\{Q\} e_{\lambda_{n}}, \text { for } D_{n+1}^{(2)}
\end{aligned}
$$

where $\epsilon=0$ except for $A_{2 n}^{(2)}, \epsilon=n$. Then the algebras $U_{q}(\hat{\mathbf{h}})$ and the space $\mathbf{C}\left[P^{\prime}\right]$ (of course $\mathbf{C}[P]$ ) act on the spaces $W_{i}$ canonically by extending their respective actions component-wise. We also define the degree action by

$$
d . f \otimes e^{\alpha} e_{\beta}=\left(-\sum_{j=1}^{k} m_{j}-\sum_{j=1}^{l} n_{j}-\frac{\langle\beta \mid \beta\rangle}{2}+\sum_{s=0}^{r-1} \frac{\left\langle\alpha_{i}^{\prime} \mid \sigma^{s}\left(\alpha_{i}^{\prime}\right)\right\rangle}{2}\right) f \otimes e^{\alpha} e_{\beta},
$$

where $f \otimes e^{\alpha} e_{\beta}=a_{i_{1}}\left(-m_{1}\right) \cdots a_{i_{k}}\left(-m_{k}\right) \otimes e^{\alpha} e_{\beta} \in W_{i}$. For convenience we write $\lambda_{\epsilon}=0$.

Proposition 3.1. The space $W_{i}(i=\epsilon, 1, n)$ is the irreducible representation $V\left(\Lambda_{i}\right)$ of the twisted quantum affine algebra $U_{q}\left(X_{N}^{(r)}\right)$ under the action

$$
\begin{gathered}
\gamma \mapsto q, K_{j} \mapsto q^{\frac{p_{i}}{r} \partial_{\alpha_{j}}}, a_{j}(k) \mapsto a_{j}(k) \quad(1 \leq j \leq n), \\
x_{i}^{ \pm}(z) \mapsto X_{i}^{ \pm}(z)=\sqrt{p_{i}} \exp \left( \pm \sum_{k=1}^{\infty} \frac{a_{i}(-k)}{\left[k / d_{i}\right]_{i}} q^{\mp k / 2} z^{k}\right) \exp \left(\mp \sum_{k=1}^{\infty} \frac{a_{i}(k)}{\left[k / d_{i}\right]_{i}} q^{\mp k / 2} z^{-k}\right) \\
\times e^{ \pm \alpha_{i}} z^{ \pm \frac{p_{i}}{r} \partial_{\alpha_{i}}+1},
\end{gathered}
$$

where $p_{i}=1$ or $r$ according to $\sigma(i) \neq i$ or $i$, and the degree operator $d$ acts by (3.9). The highest weight vectors are respectively

$$
\left|\Lambda_{i}\right\rangle=1 \otimes e_{\lambda_{i}} \otimes 1, \quad i=\epsilon, 1, n
$$

for appropriate cases.

Proof. The detail of the proof is given in [9] for the case of $\lambda_{\epsilon}$, and the proof in the cases of $\lambda_{1}$ for $A_{2 n-1}^{(2)}$ and $\lambda_{n}$ for $D_{n+1}^{(2)}$ is exactly the same as that of $\lambda_{\epsilon}$ by the standard shift method of the highest weight vectors. The irreducibility is easily shown by using the theory of the quantum $Z$-algebra operators given by the first author [10].

\section{Realization of the LeVEl one Vertex operators}

We first recall the notion of Frenkel-Reshetikhin vertex operators [5]. Let $V$ be a finite dimensional representation of the derived quantum affine Lie algebra $U_{q}^{\prime}\left(X_{N}^{(r)}\right)$ with the associated affinization space $V_{z}$ (cf. section 2). Let $V(\lambda)$ and $V(\mu)$ be two integrable highest weight irreducible representations of $U_{q}\left(X_{N}^{(r)}\right)$. The type I (resp. 
type II) vertex operator is the $U_{q}\left(X_{N}^{(r)}\right)$-intertwining operator: $V(\lambda) \longrightarrow V(\mu) \hat{\otimes} V_{z}$ (resp. $\left.V(\lambda) \longrightarrow V_{z} \hat{\otimes} V(\mu)\right)$. For simplicity we will compute the intertwining operators for the derived subalgebra $U_{q}^{\prime}\left(X_{N}^{(r)}\right)$, which differ only by a power of $z$ from those for $U_{q}\left(X_{N}^{(r)}\right)$. Also we will omit ^ in the tensor notation.

The existence of vertex operators is given by the fundamental fact [5] (cf. [2]) (true for both types, though stated for type I):

$$
\begin{aligned}
\operatorname{Hom}_{U_{q}\left(X_{N}^{(r)}\right)}\left(V(\lambda), V(\mu) \otimes V_{z}\right) \simeq & \{v \in V \mid w t(v)=\lambda-\mu \bmod \delta \text { and } \\
& \left.e_{i}^{\left\langle\mu, h_{i}\right\rangle+1} v=0 \text { for } i=0, \cdots, n\right\},
\end{aligned}
$$

where the isomorphism is defined by sending an element $\Phi \in H_{o m_{U_{q}}}(V(\lambda), V(\mu) \otimes$ $V_{z}$ ) to an element $v \in V$ such that

$$
\Phi|\lambda\rangle=|\mu\rangle \otimes v+(\text { higher terms in the powers of } z) .
$$

For the evaluation module $V_{z}$, we define the components of the vertex operator $\Phi_{\lambda}^{\mu V}: V(\lambda) \longrightarrow V(\mu) \otimes V_{z}$ by

$$
\Phi_{\lambda}^{\mu V}(z)|u\rangle=\sum_{j=1}^{n} \Phi_{\lambda j}^{\mu V}(z)|u\rangle \otimes v_{j}+\Phi_{\lambda 0}^{\mu V}(z)|u\rangle \otimes v_{0}+\sum_{j=1}^{n} \Phi_{\lambda \bar{j}}^{\mu V}(z)|u\rangle \otimes v_{\bar{j}}
$$

for $|u\rangle \in V(\lambda)$. The components of type II vertex operators are defined similarly.

We also consider the intertwining operators of modules of the following form:

$$
\Phi_{\lambda V}^{\mu}(z): V(\lambda) \otimes V_{z} \longrightarrow V(\mu) \otimes \mathbf{C}\left[z, z^{-1}\right]
$$

by means of the vertex operators with respect to the dual space $V_{z}^{*}$ :

$$
\Phi_{\lambda V}^{\mu}(z)\left(|v\rangle \otimes v_{i}\right)=\Phi_{\lambda i}^{\mu V^{*}}(z)|v\rangle
$$

for $|v\rangle \in V(\lambda)$ and $i=1, \cdots, n, 0, \bar{n}, \cdots, \overline{1}$. In other words, the vertex operator $\Phi_{\lambda i}^{\mu V^{*}}(z)$ can be defined directly as in the case of $\Phi_{\lambda i}^{\mu V}(z)$.

From the above fact we determine that the level one vertex operators exist only in the following cases: between $V\left(\Lambda_{0}\right)$ and $V\left(\Lambda_{1}\right)$ for $A_{2 n-1}^{(2)}$; between $V\left(\Lambda_{i}\right)$ and itself, $i=0, n$ for $D_{n+1}^{(2)}$; between $V\left(\Lambda_{n}\right)$ and itself for $A_{2 n}^{(2)}$; between $V\left(\Lambda_{0}\right)$ and itself for $D_{4}^{(3)}$.

We take the following normalization.

For $A_{2 n-1}^{(2)}$,

$$
\begin{aligned}
\Phi_{\Lambda_{0} V}^{\Lambda_{1} V}(z)\left|\Lambda_{0}\right\rangle & =\left|\Lambda_{1}\right\rangle \otimes v_{\overline{1}}+\text { higher terms in } z, \\
\Phi_{\Lambda_{1} V}^{\Lambda_{1}}(z)\left|\Lambda_{1}\right\rangle & =\left|\Lambda_{0}\right\rangle \otimes v_{1}+\text { higher terms in } z, \\
\Phi_{\Lambda_{0} V^{*}}^{\Lambda_{1}}(z)\left|\Lambda_{0}\right\rangle & =\left|\Lambda_{1}\right\rangle \otimes v_{1}^{*}+\text { higher terms in } z, \\
\Phi_{\Lambda_{1}}^{\Lambda_{0} V^{*}}(z)\left|\Lambda_{1}\right\rangle & =\left|\Lambda_{0}\right\rangle \otimes v_{\overline{1}}^{*}+\text { higher terms in } z .
\end{aligned}
$$

For $D_{n+1}^{(2)}$,

$$
\begin{aligned}
\Phi_{\Lambda_{0} V}^{\Lambda_{0} V}(z)\left|\Lambda_{0}\right\rangle & =\left|\Lambda_{0}\right\rangle \otimes v_{\overline{0}}+\text { higher terms in } z, \\
\Phi_{\Lambda_{0} V^{*}}^{\Lambda_{0}}(z)\left|\Lambda_{0}\right\rangle & =\left|\Lambda_{0}\right\rangle \otimes v_{0}^{*}+\text { higher terms in } z, \\
\Phi_{\Lambda_{n} V}^{\Lambda_{n}}(z)\left|\Lambda_{n}\right\rangle & =\left|\Lambda_{n}\right\rangle \otimes v_{0}+\text { higher terms in } z, \\
\Phi_{\Lambda_{n} V^{*}}^{\Lambda_{n}}(z)\left|\Lambda_{n}\right\rangle & =\left|\Lambda_{n}\right\rangle \otimes v_{0}^{*}+\text { higher terms in } z .
\end{aligned}
$$


For $A_{2 n}^{(2)}$

$$
\begin{aligned}
\Phi_{\Lambda_{n} V}^{\Lambda_{n} V}(z)\left|\Lambda_{n}\right\rangle & =\left|\Lambda_{n}\right\rangle \otimes v_{0}+\text { higher terms in } z \\
\Phi_{\Lambda_{n}}^{\Lambda_{n} V^{*}}(z)\left|\Lambda_{n}\right\rangle & =\left|\Lambda_{n}\right\rangle \otimes v_{0}^{*}+\text { higher terms in } z
\end{aligned}
$$

For $D_{4}^{(3)}$

$$
\begin{aligned}
\Phi_{\Lambda_{0} V}^{\Lambda_{0} V}(z)\left|\Lambda_{0}\right\rangle & =\left|\Lambda_{0}\right\rangle \otimes v_{\overline{0}}+\text { higher terms in } z, \\
\Phi_{\Lambda_{0} V^{*}}^{\Lambda_{0}}(z)\left|\Lambda_{0}\right\rangle & =\left|\Lambda_{0}\right\rangle \otimes v_{\overline{0}}^{*}+\text { higher terms in } z .
\end{aligned}
$$

Type II vertex operators assume similar normalization.

By the intertwining property it is easy to see the following determination relations.

Proposition 4.1. The vertex operator $\Phi(z)$ of type $I$ with respect to $V_{z}$ is determined by its component $\Phi_{\overline{1}}(z)$. With respect to $V_{z}^{*}$, the vertex operator $\Phi^{*}(z)$ of type I is determined by $\Phi_{1}^{*}(z)$. More explicitly, we have for $A_{2 n-1}^{(2)}$

$$
\begin{aligned}
\Phi_{i}(z) & =\left[\Phi_{i+1}(z), f_{i}\right]_{q} \quad \text { for } \quad i=1, \cdots, n-1, \\
\Phi_{\overline{i+1}}(z) & =\left[\Phi_{\bar{i}}(z), f_{i}\right]_{q} \quad \text { for } i=1, \cdots, n-1, \\
{\left[f_{i}, \Phi_{i}(z)\right]_{q} } & =0, \quad\left[f_{i}, \Phi_{\overline{i+1}}(z)\right]_{q}=0, \\
{\left[f_{i}, \Phi_{j}(z)\right] } & =0, \quad \text { for } \quad j \neq i, i+1, \overline{i+1}, \bar{i} \\
\Phi_{n}(z) & =\left[\Phi_{\bar{n}}(z), f_{n}\right]_{q^{2}}, \\
{\left[f_{n}, \Phi_{n}(z)\right]_{q^{2}} } & =0, \quad\left[f_{n}, \Phi_{j}(z)\right]=0, \quad j \neq n, \bar{n}, \bar{n} ; \\
\Phi_{i+1}^{*}(z) & =\left[f_{i}, \Phi_{i}^{*}(z)\right]_{q^{-1}} \quad \text { for } \quad i=1, \cdots, n-1, \\
\Phi_{\bar{i}}^{*}(z) & =\left[f_{i}, \Phi_{\overline{i+1}}^{*}(z)\right]_{q^{-1}} \quad \text { for } \quad i=1, \cdots, n-1, \\
{\left[\Phi_{i+1}^{*}(z), f_{i}\right]_{q^{-1}} } & =0, \quad\left[\Phi_{\bar{i}}^{*}(z), f_{i}\right]_{q^{-1}}=0, \\
\Phi_{\bar{n}}^{*}(z) & =\left[f_{n}, \Phi_{n}^{*}(z)\right]_{q^{-2}}, \quad \quad \quad \quad \quad j \neq i, i+1, \overline{i+1}, \bar{i}, \\
{\left[\Phi_{j}^{*}(z), f_{j}\right] } & =0, \quad \text { for } \quad j \neq n, \bar{n}, \bar{n} .
\end{aligned}
$$

for $D_{n+1}^{(2)}$,

$$
\begin{aligned}
\Phi_{i}(z) & =\left[\Phi_{i+1}(z), f_{i}\right]_{q^{2}} \quad \text { for } \quad i=1, \cdots, n-1, \\
\Phi_{\overline{i+1}}(z) & =\left[\Phi_{\bar{i}}(z), f_{i}\right]_{q^{2}} \quad \text { for } \quad i=1, \cdots, n-1, \\
{\left[f_{i}, \Phi_{i}(z)\right]_{q^{2}} } & =0, \quad\left[f_{i}, \Phi_{\overline{i+1}}(z)\right]_{q^{2}}=0, \\
{\left[f_{i}, \Phi_{j}(z)\right] } & =0, \quad \text { for } \quad j \neq i, i+1, \overline{i+1}, \bar{i}, \\
\Phi_{n}(z) & =\left[\Phi_{0}(z), f_{n}\right], \\
\Phi_{0}(z) & =[2]^{-1}\left[\Phi_{\bar{n}}(z), f_{n}\right]_{q^{2}}, \\
{\left[f_{n}, \Phi_{n}(z)\right]_{q^{2}} } & =0, \quad\left[f_{n}, \Phi_{j}(z)\right]=0, \quad j \neq n, 0, \bar{n}, \\
\Phi_{\overline{0}}(z) & =[2]^{-1} z\left[\Phi_{1}(z), f_{0}\right]_{q^{2}}, \quad \Phi_{\overline{1}}(z)=z\left[\Phi_{\overline{0}}(z), f_{0}\right], \\
{\left[\Phi_{\overline{1}}(z), f_{0}\right]_{q^{2}} } & =0, \quad\left[\Phi_{j}(z), f_{0}\right]=0, \quad \text { for } j \neq 1, \overline{0}, \overline{1} ; \\
\Phi_{i+1}^{*}(z) & =\left[f_{i}, \Phi_{i}^{*}(z)\right]_{q^{-2}} \quad \text { for } \quad i=1, \cdots, n-1, \\
\Phi_{\bar{i}}^{*}(z) & =\left[f_{i}, \Phi_{\overline{i+1}}^{*}(z)\right]_{q^{-2}} \quad \text { for } \quad i=1, \cdots, n-1,
\end{aligned}
$$




$$
\begin{aligned}
{\left[\Phi_{i+1}^{*}(z), f_{i}\right]_{q^{-2}} } & =0, \quad\left[\Phi_{\bar{i}}^{*}(z), f_{i}\right]_{q^{-2}}=0, \\
\Phi_{0}^{*}(z) & =\left[f_{n}, \Phi_{n}^{*}(z)\right]_{q^{-2}}, \quad \Phi_{\bar{n}}^{*}(z)=[2]^{-1}\left[f_{n}, \Phi_{0}^{*}(z)\right], \\
{\left[\Phi_{j}^{*}(z), f_{i}\right] } & =0, \quad \text { for } \quad j \neq i, i+1, \overline{i+1}, \bar{i} \\
{\left[f_{n}, \Phi_{\bar{n}}^{*}(z)\right]_{q^{-2}} } & =0, \quad\left[\Phi_{j}^{*}(z), f_{n}\right]=0, \quad j \neq n, 0, \bar{n}, \bar{n} \\
\Phi_{1}^{*}(z) & =[2]^{-1} z\left[f_{0}, \Phi_{\overline{0}}^{*}(z)\right], \quad \Phi_{\overline{0}}^{*}(z)=z\left[f_{0}, \Phi_{\overline{1}}^{*}(z)\right]_{q^{-2}}, \\
{\left[\Phi_{1}^{*}(z), f_{0}\right]_{q^{-2}} } & =0, \quad\left[\Phi_{j}^{*}(z), f_{0}\right]=0, \quad \text { for } j \neq 1, \overline{0}, \overline{1} .
\end{aligned}
$$

for $A_{2 n}^{(2)}$,

$$
\begin{aligned}
\Phi_{i}(z) & =\left[\Phi_{i+1}(z), f_{i}\right]_{q} \quad \text { for } \quad i=1, \cdots, n-1, \\
\Phi_{\overline{i+1}}(z) & =\left[\Phi_{\bar{i}}(z), f_{i}\right]_{q} \quad \text { for } \quad i=1, \cdots, n-1, \\
{\left[f_{i}, \Phi_{i}(z)\right]_{q} } & =0, \quad\left[f_{i}, \Phi_{\overline{i+1}}(z)\right]_{q}=0, \\
{\left[f_{i}, \Phi_{j}(z)\right] } & =0, \quad \text { for } \quad j \neq i, i+1, \overline{i+1}, \bar{i}, \\
\Phi_{n}(z) & =\left[\Phi_{0}(z), f_{n}\right], \\
\Phi_{0}(z) & =[2]_{n}^{-1}\left[\Phi_{\bar{n}}(z), f_{n}\right]_{q}, \\
{\left[f_{n}, \Phi_{n}(z)\right]_{q} } & =0, \quad\left[f_{n}, \Phi_{j}(z)\right]=0, \quad j \neq n, \bar{n}, \bar{n}, \\
\Phi_{\overline{1}}(z) & =z\left[\Phi_{1}(z), f_{0}\right]_{q^{2}}, \\
{\left[f_{0}, \Phi_{\overline{1}}(z)\right]_{q^{2}} } & =0, \quad\left[f_{0}, \Phi_{j}(z)\right]=0, \quad j \neq 1, \overline{1}, \\
\Phi_{i+1}^{*}(z) & =\left[f_{i}, \Phi_{i}^{*}(z)\right]_{q^{-1}} \quad \text { for } \quad i=1, \cdots, n-1, \\
\Phi_{\bar{i}}^{*}(z) & =\left[f_{i}, \Phi_{\overline{i+1}}^{*}(z)\right]_{q^{-1}} \quad \text { for } \quad i=1, \cdots, n-1, \\
{\left[\Phi_{i+1}^{*}(z), f_{i}\right]_{q^{-1}} } & =0, \quad\left[\Phi_{\bar{i}}^{*}(z), f_{i}\right]_{q^{-1}}=0, \\
\Phi_{\bar{n}}^{*}(z) & =[2]_{n}^{-1}\left[f_{n}, \Phi_{0}^{*}(z)\right], \quad \Phi_{0}^{*}(z)=\left[f_{n}, \Phi_{n}^{*}(z)\right]_{q^{-1}}, \\
{\left[\Phi_{j}^{*}(z), f_{i}\right] } & =0, \quad \text { for } \quad j \neq i, i+1, \overline{i+1}, \bar{i}, \\
{\left[\Phi_{n}^{*}(z), f_{n}\right]_{q^{-1}} } & =0, \quad\left[\Phi_{j}^{*}(z), f_{n}\right]=0, \quad j \neq n, \bar{n}, \\
\Phi_{1}^{*}(z) & =z\left[f-0, \Phi_{\overline{1}}^{*}(z)\right]_{q^{-2}}, \quad \\
{\left[\Phi_{1}^{*}(z), f_{0}\right]_{q^{-2}} } & =0, \quad\left[\Phi_{j}^{*}(z), f_{0}\right]_{q^{-2}}=0, \quad \text { for } \quad j \neq 1, \overline{1}, \bar{n},
\end{aligned}
$$

for $D_{4}^{(3)}$,

$$
\begin{aligned}
\Phi_{1}(z) & =\left[\Phi_{2}(z), f_{1}\right]_{q},\left[f_{1}, \Phi_{1}(z)\right]_{q}=0,\left[f_{1}, \Phi_{3}(z)\right]_{q^{2}}=0, \\
\Phi_{3}(z) & =\left[\Phi_{0}(z), f_{1}\right], \Phi_{\overline{0}}(z)+[2] \Phi_{0}(z)=\left[\Phi_{\overline{3}}(z), f_{1}\right]_{q^{2}}, \\
\Phi_{\overline{2}}(z) & =\left[\Phi_{\overline{1}}(z), f_{1}\right]_{q},\left[f_{1}, \Phi_{\overline{2}}(z)\right]=0,\left[f_{1}, \Phi_{\overline{0}}(z)\right]=0 \\
\Phi_{2}(z) & =\left[\Phi_{3}(z), f_{2}\right]_{q^{3}}, \Phi_{\overline{3}}(z)=\left[\Phi_{\overline{2}}(z), f_{2}\right]_{q^{3}}, \\
{\left[f_{2}, \Phi_{2}(z)\right]_{q^{3}} } & =0,\left[f_{2}, \Phi_{\overline{3}}(z)\right]_{q^{3}}=0, \\
{\left[f_{2}, \Phi_{j}(z)\right] } & =0, \quad \text { for } j=1,0, \overline{1}, \overline{0} \\
\Phi_{\overline{3}}(z) & =z\left[\Phi_{2}(z), f_{0}\right]_{q}, \Phi_{0}(z)+[2] \Phi_{\overline{0}}(z)=z\left[\Phi_{1}(z), f_{0}\right]_{q^{2}}, \\
\Phi_{\overline{2}}(z) & =z\left[\Phi_{3}(z), f_{0}\right]_{q}, \Phi_{\overline{1}}(z)=z\left[\Phi_{\overline{0}}(z), f_{0}\right], \\
{\left[f_{0}, \Phi_{j}(z)\right]_{q} } & =0, \quad \text { for } j=\overline{2}, \overline{3} \\
{\left[f_{0}, \Phi_{\overline{1}}(z)\right]_{q^{2}} } & =0, \quad\left[f_{0}, \Phi_{0}(z)\right]=0
\end{aligned}
$$




$$
\begin{aligned}
\Phi_{2}^{*}(z) & =\left[f_{1}, \Phi_{1}^{*}(z)\right]_{q^{-1}},\left[\Phi_{\overline{1}}^{*}(z), f_{1}\right]_{q^{-1}}=0, \quad\left[\Phi_{\overline{3}}^{*}(z), f_{1}\right]_{q^{-2}}=0, \\
\Phi_{3}^{*}(z) & =\left[f_{1}, \Phi_{\overline{0}}^{*}(z)\right],[2] \Phi_{\frac{3}{3}}^{*}(z)=\left[f_{1}, \Phi_{0}^{*}(z)\right], \\
\Phi_{\overline{1}}^{*}(z) & =\left[f_{1}, \Phi_{\overline{2}}^{*}(z)\right]_{q^{-1}},\left[\Phi_{2}^{*}(z), f_{1}\right]_{q^{-1}}=0, \\
\Phi_{3}^{*}(z) & =\left[f_{2}, \Phi_{2}^{*}(z)\right]_{q^{-3}}, \Phi_{\overline{2}}^{*}(z)=\left[f_{2}, \Phi_{\overline{3}}^{*}(z)\right]_{q^{-3}}, \\
{\left[\Phi_{\overline{2}}^{*}(z), f_{2}\right]_{q^{-3}} } & =0, \quad\left[\Phi_{3}^{*}(z), f_{2}\right]_{q^{-3}}=0, \\
{\left[\Phi_{j}^{*}(z), f_{2}\right] } & =0, \quad \text { for } j=1,0, \overline{1}, \overline{0}, \\
\Phi_{3}^{*}(z) & =z\left[f_{0}, \Phi_{\overline{2}}(z)\right]_{q^{-1}},[2] \Phi_{1}^{*}(z)=z\left[f_{0}, \Phi_{\overline{0}}^{*}(z)\right], \\
\Phi_{2}^{*}(z) & =z\left[f_{0}, \Phi_{\overline{3}}^{*}(z)\right]_{q^{-1}}, \Phi_{\overline{0}}^{*}(z)=z\left[f_{0}, \Phi_{\overline{1}}^{*}(z)\right]_{q^{-2}}, \\
{\left[\Phi_{j}^{*}(z), f_{0}\right]_{q^{-1}} } & =0, \quad \text { for } j=2,3, \\
\Phi_{1}^{*}(z) & =z\left[f_{0}, \Phi_{0}^{*}(z)\right], \quad\left[\Phi_{1}^{*}(z), f_{0}\right]_{q^{-2}}=0 .
\end{aligned}
$$

Proposition 4.2. Let $\tilde{\Phi}(z)$ be a type II vertex operator with respect to $V_{z}: V(\lambda) \rightarrow$ $V_{z} \otimes V(\mu)$. Then $\tilde{\Phi}(z)$ is determined by the component $\tilde{\Phi}_{1}(z)$, and with respect to $V_{z}^{*}$ the vertex operator $\Phi^{*}(z)$ is determined by its component $\Phi_{\overline{1}}^{*}(z)$. More precisely, with respect to $V_{z}$, we have

for $A_{2 n-1}^{(1)}$,

$$
\begin{aligned}
\Phi_{i+1}(z) & =\left[\Phi_{i}(z), e_{i}\right]_{q} \quad i=1, \cdots, n-1, \\
\Phi_{\bar{i}}(z) & =\left[\Phi_{\overline{i+1}}(z), e_{i}\right]_{q} \quad i=1, \cdots, n-1, \\
{\left[e_{i}, \Phi_{i+1}(z)\right]_{q} } & =0, \quad\left[e_{i}, \Phi_{\bar{i}}\right]_{q}=0, \\
{\left[e_{i}, \Phi_{j}(z)\right] } & =0 \quad \text { for } j \neq i, i+1, \overline{i+1}, \bar{i} \\
\Phi_{\bar{n}}(z) & =\left[\Phi_{n}(z), e_{n}\right]_{q^{2}}, \\
{\left[e_{n}, \Phi_{\bar{n}}(z)\right]_{q^{2}} } & =0, \quad\left[e_{n}, \Phi_{j}(z)\right]=0 \quad \text { for } j \neq n, \bar{n} ; \\
\Phi_{i}^{*}(z) & =q^{2}\left[e_{i}, \Phi_{i+1}^{*}(z)\right]_{q^{-1}} \quad \text { for } i=1, \cdots, n-1, \\
\Phi_{\overline{i+1}}^{*}(z) & =q^{2}\left[e_{i}, \Phi_{\bar{i}}^{*}(z)\right]_{q^{-1}} \quad \text { for } i=1, \cdots, n-1, \\
{\left[\Phi_{i}^{*}(z), e_{i}\right]_{q^{-1}} } & =0, \quad\left[\Phi_{\overline{i+1}}^{*}(z), e_{i}\right]_{q^{-1}}=0, \\
{\left[\Phi_{j}^{*}(z), e_{i}\right] } & =0 \quad \text { for } j \neq i, i+1, \overline{i+1}, \bar{i}, \\
\Phi_{n}^{*}(z) & =q^{4}\left[e_{n}, \Phi_{\bar{n}}^{*}(z)\right]_{q^{-2}}, \quad\left[\Phi_{j}^{*}(z), e_{n}\right]=0 \quad \text { for } j \neq n, 0, \bar{n} .
\end{aligned}
$$

for $D_{n+1}^{(2)}$,

$$
\begin{aligned}
\Phi_{i+1}(z) & =\left[\Phi_{i}(z), e_{i}\right]_{q^{2}} \quad \text { for } \quad i=1, \cdots, n-1, \\
\Phi_{\bar{i}}(z) & =\left[\Phi_{\overline{i+1}}(z), e_{i}\right]_{q^{2}} \quad \text { for } \quad i=1, \cdots, n-1, \\
{\left[e_{i}, \Phi_{i+1}(z)\right]_{q^{2}} } & =0, \quad\left[e_{i}, \Phi_{\bar{i}}(z)\right]_{q^{2}}=0, \\
{\left[e_{i}, \Phi_{j}(z)\right] } & =0, \quad \text { for } \quad j \neq i, i+1, \overline{i+1}, \bar{i} \\
\Phi_{\bar{n}}(z) & =\left[\Phi_{0}(z), e_{n}\right], \\
\Phi_{0}(z) & =[2]^{-1}\left[\Phi_{n}(z), e_{n}\right]_{q^{2}}, \\
{\left[e_{n}, \Phi_{\bar{n}}(z)\right]_{q^{2}} } & =0, \quad\left[e_{n}, \Phi_{j}(z)\right]=0, \quad j \neq n, 0, \bar{n}, \bar{n}, \\
\Phi_{\overline{0}}(z) & =[2]^{-1} z^{-1}\left[\Phi_{\overline{1}}(z), e_{0}\right]_{q^{2}}, \quad \Phi_{1}(z)=z^{-1}\left[\Phi_{\overline{0}}(z), e_{0}\right], \\
{\left[e_{0}, \Phi_{1}(z)\right]_{q^{2}} } & =0, \quad\left[\Phi_{j}(z), e_{0}\right]=0, \quad \text { for } j \neq 1, \overline{0}, \overline{1},
\end{aligned}
$$




$$
\begin{aligned}
\Phi_{i}^{*}(z) & =q^{4}\left[e_{i}, \Phi_{i}^{*}(z)\right]_{q^{-2}} \quad \text { for } \quad i=1, \cdots, n-1, \\
\Phi_{\bar{i}+1}^{*}(z) & =q^{4}\left[e_{i}, \Phi_{\overline{\bar{l}}}^{*}(z)\right]_{q^{-2}} \quad \text { for } \quad i=1, \cdots, n-1, \\
{\left[\Phi_{i}^{*}(z), e_{i}\right]_{q^{-2}} } & =0, \quad\left[\Phi_{\bar{i}+1}^{*}(z), e_{i}\right]_{q^{-2}}=0, \\
\Phi_{0}^{*}(z) & =q^{2}\left[e_{n}, \Phi_{\bar{n}}^{*}(z)\right], \quad \Phi_{n}^{*}(z)=[2]^{-1}\left[e_{n}, \Phi_{0}^{*}(z)\right], \\
{\left[\Phi_{j}^{*}(z), e_{j}\right] } & =0, \quad \text { for } \quad j \neq i, i+1, \overline{i+1}, \bar{i}, \\
{\left[\Phi_{n}^{*}(z), e_{n}\right]_{q^{-2}} } & =0, \quad\left[\Phi_{j}^{*}(z), e_{n}\right]=0, \quad j \neq n, 0, \bar{n}, \bar{n}, \\
\Phi_{\overline{1}}^{*}(z) & =q^{2}[2]^{-1} z^{-1}\left[e_{0}, \Phi_{\overline{0}}^{*}(z)\right], \quad \Phi_{\overline{0}}^{*}(z)=z^{-1}\left[e_{0}, \Phi_{\overline{1}}^{*}(z)\right]_{q^{-2}}, \\
{\left[\Phi_{\overline{1}}^{*}(z), e_{0}\right]_{q^{-2}} } & =0, \quad\left[\Phi_{j}^{*}(z), e_{0}\right]=0, \quad \text { for } j \neq 1, \overline{0}, \overline{1} .
\end{aligned}
$$

for $A_{2 n}^{(2)}$,

$$
\begin{aligned}
\Phi_{i+1}(z) & =\left[\Phi_{i}(z), e_{i}\right]_{q} \quad \text { for } \quad i=1, \cdots, n-1, \\
\Phi_{\bar{i}}(z) & =\left[\Phi_{\overline{i+1}}(z), e_{i}\right]_{q} \quad \text { for } \quad i=1, \cdots, n-1, \\
{\left[e_{i}, \Phi_{i+1}(z)\right]_{q} } & =0, \quad\left[e_{i}, \Phi_{\bar{i}}(z)\right]_{q}=0, \\
{\left[e_{i}, \Phi_{j}(z)\right] } & =0, \quad \text { for } \quad j \neq i, i+1, \overline{i+1}, \bar{i}, \\
\Phi_{\bar{n}}(z) & =\left[\Phi_{0}(z), e_{n}\right], \\
\Phi_{0}(z) & =[2]_{n}^{-1}\left[\Phi_{n}(z), e_{n}\right]_{q}, \\
{\left[e_{n}, \Phi_{\bar{n}}(z)\right]_{q} } & =0, \quad\left[e_{n}, \Phi_{j}(z)\right]=0, \quad j \neq n, 0, \bar{n}, \bar{n}, \\
\Phi_{1}(z) & =z^{-1}\left[\Phi_{\overline{1}}(z), e_{0}\right]_{q^{2}}, \\
{\left[e_{0}, \Phi_{1}(z)\right]_{q^{2}} } & =0, \quad\left[e_{0}, \Phi_{j}(z)\right]=0, \quad j \neq 1, \overline{1} ; \\
\Phi_{i}^{*}(z) & =q^{2}\left[e_{i}, \Phi_{i+1}^{*}(z)\right]_{q^{-1}} \quad \text { for } i=1, \cdots, n-1, \\
\Phi_{\overline{i+1}}^{*}(z) & =q^{2}\left[e_{i}, \Phi_{\bar{i}}^{*}(z)\right]_{q^{-1}} \quad \text { for } \quad i=1, \cdots, n-1, \\
{\left[\Phi_{i}^{*}(z), e_{i}\right]_{q^{-1}} } & =0, \quad\left[\Phi_{\overline{i+1}}^{*}(z), e_{i}\right]_{q^{-1}}=0, \\
\Phi_{n}^{*}(z) & =q[2]_{n}^{-1}\left[e_{n}, \Phi_{0}^{*}(z)\right], \quad \Phi_{0}^{*}(z)=q\left[e_{n}, \Phi_{\bar{n}}^{*}(z)\right]_{q^{-1}}, \\
{\left[\Phi_{j}^{*}(z), e_{j}\right] } & =0, \quad \text { for } \quad j \neq i, i+1, \overline{i+1}, \bar{i}, \\
{\left[\Phi_{n}^{*}(z), e_{n}\right]_{q^{-1}} } & =0, \quad\left[\Phi_{j}^{*}(z), e_{n}\right]=0, \quad j \neq n, \bar{n}, \bar{n}, \\
\Phi_{\overline{1}}^{*}(z) & =q^{4}\left[e_{0}, \Phi_{1}^{*}(z)\right]_{q^{-2}}, \\
{\left[\Phi_{\overline{1}}^{*}(z), e_{0}\right]_{q^{-2}} } & =0, \quad\left[\Phi_{j}^{*}(z), e_{0}\right]=0, \quad \text { for } j \neq 1, \overline{1} .
\end{aligned}
$$

for $D_{4}^{(3)}$,

$$
\begin{aligned}
\Phi_{2}(z) & =\left[\Phi_{1}(z), e_{1}\right]_{q},[2] \Phi_{0}(z)+\Phi_{\overline{0}}(z)=\left[\Phi_{3}(z), e_{1}\right]_{q^{2}} \\
\Phi_{\overline{3}}(z) & =\left[\Phi_{0}(z), e_{1}\right], \Phi_{\overline{1}}(z)=\left[\Phi_{\overline{2}}(z), e_{1}\right]_{q}, \\
{\left[e_{1}, \Phi_{2}(z)\right]_{q} } & =0, \quad\left[e_{1}, \Phi_{\overline{3}}(z)\right]_{q^{2}}=0 \\
{\left[e_{1}, \Phi_{\overline{1}}(z)\right]_{q} } & =0,\left[e_{1}, \Phi_{\overline{0}}(z)\right]=0 \\
\Phi_{3}(z) & =\left[\Phi_{2}(z), e_{2}\right]_{q^{3}}, \Phi_{\overline{2}}(z)=\left[\Phi_{\overline{3}}(z), e_{2}\right]_{q^{3}}, \\
{\left[e_{2}, \Phi_{j}(z)\right]_{q^{3}} } & =0, \quad \text { for } j=3, \overline{2} \\
{\left[e_{2}, \Phi_{j}(z)\right] } & =0, \quad \text { for } j=1,0, \overline{1}, \overline{0}
\end{aligned}
$$




$$
\begin{aligned}
\Phi_{1}(z) & =z^{-1}\left[\Phi_{\overline{0}}(z), e_{0}\right], \Phi_{2}(z)=z^{-1}\left[\Phi_{\overline{3}}(z), e_{0}\right], \\
\Phi_{3}(z) & =z^{-1}\left[\Phi_{\overline{2}}(z), e_{0}\right]_{q}, \Phi_{0}(z)+[2] \Phi_{\overline{0}}(z)=z^{-1}\left[\Phi_{\overline{1}}(z), e_{0}\right]_{q^{2}}, \\
{\left[e_{0}, \Phi_{j}(z)\right]_{q} } & =0, \quad \text { for } j=2,3, \\
{\left[e_{0}, \Phi_{1}(z)\right]_{q^{2}} } & =0, \quad\left[f_{0}, \Phi_{0}(z)\right]=0 ; \\
\Phi_{1}^{*}(z) & =q^{2}\left[e_{1}, \Phi_{2}^{*}(z)\right]_{q^{-1}}, \Phi_{\overline{2}}^{*}(z)=q^{2}\left[e_{1}, \Phi_{\overline{1}}^{*}(z)\right]_{q^{-1}}, \\
\Phi_{0}^{*}(z) & =q^{2}\left[e_{1}, \Phi_{\overline{3}}^{*}(z)\right]_{q^{-2}}, \quad[2] \Phi_{3}^{*}(z)=q^{2}\left[e_{1}, \Phi_{0}^{*}(z)\right], \\
\Phi_{3}^{*}(z) & =q^{2}\left[e_{1}, \Phi_{\overline{0}}^{*}(z)\right], \quad\left[\Phi_{1}^{*}(z), e_{1}\right]_{q^{-1}}=0, \\
{\left[\Phi_{3}^{*}(z), e_{1}\right]_{q^{-2}} } & =0, \quad\left[\Phi_{\overline{2}}^{*}(z), e_{1}\right]_{q^{-1}}=0, \\
\Phi_{2}^{*}(z) & =q^{-6}\left[e_{2}, \Phi_{3}^{*}(z)\right]_{q^{-3}}, \Phi_{\overline{3}}^{*}(z)=q^{6}\left[e_{2}, \Phi_{\overline{2}}^{*}(z)\right]_{q^{-3}}, \\
{\left[\Phi_{j}^{*}(z), e_{2}\right]_{q^{-3}} } & =0, \quad \text { for } \quad j=2, \overline{3} \\
\Phi_{\overline{3}}^{*}(z) & =z^{-1} q^{2}\left[e_{0}, \Phi_{2}^{*}(z)\right]_{q^{-1}}, \Phi_{\overline{2}}^{*}(z)=z^{-1} q^{2}\left[e_{0}, \Phi_{3}^{*}(z)\right]_{q^{-1}}, \\
\Phi_{\overline{0}}^{*}(z) & =z^{-1} q^{2}\left[e_{0}, \Phi_{1}^{*}(z)\right]_{q^{-2}}, \\
{[2] \Phi_{\overline{1}}^{*}(z) } & =z^{-1} q^{2}\left[e_{0}, \Phi_{0}^{*}(z)\right], \quad\left[\Phi_{\overline{1}}^{*}(z), e_{0}\right]_{q^{-2}}=0, \\
{\left[\Phi_{j}^{*}(z), e_{0}\right]_{q^{-1}} } & =0, \quad \text { for } j=\overline{2}, \overline{3} .
\end{aligned}
$$

Next we determine the relations of vertex operators and Drinfeld generators.

Proposition 4.3. (a) Let $\Phi(z): V(\lambda) \longrightarrow V(\mu) \otimes V_{z}$ be a vertex operator of type $I$, where $(\lambda, \mu)=\left(\Lambda_{0}, \Lambda_{1}\right),\left(\Lambda_{1}, \Lambda_{0}\right),\left(\Lambda_{n}, \Lambda_{n}\right)$. Then we have for each $j=1, \cdots, n$ and $k \in \mathbf{N}$

$$
\begin{gathered}
{\left[\Phi_{\overline{1}}(z), X_{j}^{+}(w)\right]=0,} \\
t_{j} \Phi_{\overline{1}}(z) t_{j}^{-1}=q^{\delta_{j 1}} \Phi_{\overline{1}}(z), \\
{\left[a_{j}(k), \Phi_{\overline{1}}(z)\right]= \begin{cases}\delta_{j 1} \frac{[k]}{k} q^{\frac{4 n+3}{2}} k(-z)^{k} \Phi_{\overline{1}}(z), & \text { for } A_{2 n-1}^{(2)}, \\
\delta_{j 1} \frac{[l] 1}{l}(-1)^{n l} q^{(4 n+3) l} z^{2 l} \Phi_{\overline{1}}(z), & \text { for } k=2 l, D_{n+1}^{(2)}, \\
\delta_{j 1} \frac{[k]}{k} q^{\frac{4 n+5}{2} k}(-z)^{k} \Phi_{\overline{1}}(z), & \text { for } A_{2 n}^{(2)}, \\
\delta_{j 1} \frac{[k]}{k} q^{\frac{15}{2} k} z^{k} \Phi_{\overline{1}}(z), & \text { for } D_{4}^{(3)},\end{cases} } \\
{\left[a_{j}(-k), \Phi_{\overline{1}}(z)\right]= \begin{cases}\delta_{j 1} \frac{[k]}{k} q^{-\frac{4 n+1}{2} k}(-z)^{-k} \Phi_{\overline{1}}(z), & \text { for } A_{2 n-1}^{(2)}, \\
\delta_{j 1} \frac{\left[l_{1}\right.}{l}(-1)^{n l} q^{-(2 n+1) l} z^{-2 l} \Phi_{\overline{1}}(z), & \text { for } k=2 l, D_{n+1}^{(2)}, \\
\delta_{j 1} \frac{[k]}{k} q^{-\frac{4 n+3}{2} k}(-z)^{-k} \Phi_{\overline{1}}(z), & \text { for } A_{2 n}^{(2)}, \\
\delta_{j 1} \frac{[k]}{k} q^{-\frac{13}{2} k} z^{-k} \Phi_{\overline{1}}(z), & \text { for } A_{2 n}^{(2)} .\end{cases} }
\end{gathered}
$$

(b) If $\Phi(z)$ is a vertex operator of type I associated with $V_{z}^{*}$, then

$$
\begin{aligned}
{\left[\Phi_{1}^{*}(z), X_{j}^{+}(w)\right] } & =0, \\
t_{j} \Phi_{1}^{*}(z) t_{j}^{-1} & =q^{\delta_{j 1}} \Phi_{1}^{*}(z), \\
{\left[a_{j}(k), \Phi_{1}^{*}(z)\right] } & = \begin{cases}\delta_{j 1} \frac{[k]}{k} q^{\frac{3 k}{2}} z^{k} \Phi_{1}^{*}(z), & \text { for } A_{2 n-1}^{(2)}, A_{2 n}^{(2)}, D_{4}^{(3)}, \\
\delta_{j 1} \frac{[l] 1}{l}(-1)^{n l} q^{3 l} z^{2 l} \Phi_{1}^{*}(z), & \text { for } k=2 l, D_{n+1}^{(2)},\end{cases} \\
{\left[a_{j}(-k), \Phi_{1}^{*}(z)\right] } & = \begin{cases}\delta_{j 1} \frac{[k]}{k} q^{-\frac{k}{2}} z^{-k} \Phi_{1}^{*}(z), & \text { for } A_{2 n-1}^{(2)}, A_{2 n}^{(2)}, D_{4}^{(3)}, \\
\delta_{j 1} \frac{[l] 1}{l}(-1)^{n l} q^{-l} z^{-2 l} \Phi_{1}^{*}(z), & \text { for } k=2 l, D_{n+1}^{(2)} .\end{cases}
\end{aligned}
$$


(c) If $\Phi(z)$ is a vertex operator of type II associated with $V_{z}$, then

$$
\begin{aligned}
{\left[\Phi_{1}(z), X_{j}^{-}(w)\right] } & =0, \\
t_{j} \Phi_{1}(z) t_{j}^{-1} & =q^{-\delta_{j 1}} \Phi_{1}(z), \\
{\left[a_{j}(k), \Phi_{1}(z)\right] } & = \begin{cases}-\delta_{j} \frac{[k]}{k} q^{\frac{k}{2}} z^{k} \Phi_{1}(z), & \text { for } A_{2 n-1}^{(2)}, A_{2 n}^{(2)}, D_{4}^{(3)}, \\
-\delta_{j 1} \frac{[l] 1}{l}(-1)^{n l} q^{l} z^{2 l} \Phi_{1}(z), & \text { for } k=2 l D_{n+1}^{(2)},\end{cases} \\
{\left[a_{j}(-k), \Phi_{1}(z)\right] } & = \begin{cases}-\delta_{j 1} \frac{[k]}{k} q^{-\frac{3 k}{2}} z^{-k} \Phi_{1}(z), & \text { for } A_{2 n-1}^{(2)}, A_{2 n}^{(2)}, D_{4}^{(3)}, \\
-\delta_{j 1} \frac{[l] 1}{l}(-1)^{n l} q^{-3 l} z^{2 l} \Phi_{1}(z), & \text { for } k=2 l, D_{n+1}^{(2)} .\end{cases}
\end{aligned}
$$

(d) If $\Phi^{*}(z)$ is a vertex operator of type II associated with $V_{z}^{*}$, then

$$
\begin{aligned}
& {\left[\Phi_{\overline{1}}^{*}(z), X_{j}^{-}(w)\right]=} 0, \\
& t_{j} \Phi_{\overline{1}}^{*}(z) t_{j}^{-1}= q^{-\delta_{j 1}} \Phi_{\overline{1}}^{*}(z), \\
& {\left[a_{j}(k), \Phi_{\overline{1}}^{*}(z)\right]=} \begin{cases}-\delta_{j 1} \frac{[k]}{k} q^{-\frac{4 n-1}{2} k}(-z)^{k} \Phi_{1}^{*}(z), & \text { for } A_{2 n-1}^{(2)}, \\
-\delta_{j 1} \frac{[l] 1}{l}(-1)^{n l} q^{-(4 n-1) l} z^{2 l} \Phi_{\overline{1}}^{*}(z), & \text { for } k=2 l, D_{n+1}^{(2)}, \\
-\delta_{j 1} \frac{[k]}{k} q^{-\frac{4 n+1}{2} k}(-z)^{k} \Phi_{\overline{1}}^{*}(z), & \text { for } A_{2 n}^{(2)}, \\
-\delta_{j 1} \frac{[k]}{k} q^{-\frac{11}{2} k} z^{k} \Phi_{\overline{1}}^{*}(z), & \text { for } D_{4}^{(3)},\end{cases} \\
& {\left[a_{j}(-k), \Phi_{\overline{1}}^{*}(z)\right]= \begin{cases}-\delta_{j 1} \frac{[k]}{k} q^{\frac{4 n-3}{2} k}(-z)^{-k} \Phi_{\overline{1}}^{*}(z), & \text { for } A_{2 n-1}^{(2)}, \\
-\delta_{j 1} \frac{[l] 1}{l}(-1)^{n l} q^{(2 n-3) l} z^{-2 l} \Phi_{\overline{1}}^{*}(z), & \text { for } k=2 l, D_{n+1}^{(2)}, \\
-\delta_{j 1} \frac{[k]}{k} q^{\frac{4 n-1}{2} k}(-z)^{-k} \Phi_{\overline{1}}^{*}(z), & \text { for } A_{2 n}^{(2)}, \\
-\delta_{j 1} \frac{[k]}{k} q^{\frac{9}{2} k} z^{-k} \Phi_{1}^{*}(z), & \text { for } D_{4}^{(3)} .\end{cases} }
\end{aligned}
$$

We introduce the auxiliary element $a_{\overline{1}}(k) \in U_{q}\left(\hat{\mathbf{h}}^{-}\right)\left(k \in \mathbf{Z}^{\times}\right)$such that

$$
\left[a_{i}(k), a_{\overline{1}}(l)\right]=\delta_{i 1} \delta_{k,-l},
$$

where $k$ and $l$ are even integers in the case of $D_{n+1}^{(2)}$. We determine that

$$
\begin{aligned}
& A_{2 n-1}^{(2)}: \quad a_{\overline{1}}(k)=\frac{-k}{[k]^{2}}\left(\sum_{i=1}^{n-1} \frac{q^{(n-i) k}+(-1)^{k} q^{-(n-i) k}}{q^{n k}+(-1)^{k} q^{-n k}} a_{i}(k)\right. \\
& \left.+\frac{q+q^{-1}}{q^{n k}+q^{-n k}} a_{n}(k)\right) \\
& D_{n+1}^{(2)}: \quad a_{\overline{1}}(2 k)=f r a c-k[k]_{1}^{2}\left(\sum_{i=1}^{n-1} \frac{q^{2(n-i) k}+q^{-2(n-i) k}}{q^{2 n k}+q^{-2 n k}} a_{i}(2 k)\right. \\
& \left.+\frac{2}{[2]\left(q^{2 n k}+q^{-2 n k}\right)} a_{n}(2 k)\right) \text {, } \\
& A_{2 n}^{(2)}: \quad a_{\overline{1}}(k)=\frac{-k}{[k]^{2}}\left(\sum_{i=1}^{n-1} \frac{q^{(2 n-2 i+1) k / 2}+(-1)^{k} q^{-(2 n-2 i+1) k / 2}}{q^{(2 n+1) k / 2}+(-1)^{k} q^{-(2 n+1) k / 2}} a_{i}(k)\right. \\
& \left.+\frac{q^{k / 2}+(-1)^{k} q^{-k / 2}}{\left(q^{1 / 2}+q^{-1 / 2}\right)\left(q^{(2 n+1) k / 2}+(-1)^{k} q^{-(2 n+1) k / 2}\right)} a_{n}(k)\right),
\end{aligned}
$$




$$
\begin{aligned}
D_{4}^{(3)}: \quad a_{\overline{1}}(k)= & \frac{-k}{[k][2 k]}\left(\frac{\prod_{i=0}^{2}\left(q^{k}+\omega^{i k} q^{-k}\right)}{q^{3 k}+q^{-3 k}} a_{1}(k)\right. \\
& \left.+\frac{\left(q^{k}+q^{-k}\right)^{2}}{q^{3 k}+q^{-3 k}} a_{2}(k)\right)
\end{aligned}
$$

where $a_{n}(k)=0$ if $k \neq 0 \bmod r$ for $A_{2 n-1}^{(2)}$ and $D_{4}^{(3)}$ by definition.

We now state our main results on the realization of vertex operators for the level one $U_{q}\left(X_{N}^{(r)}\right)$-modules. We only need to determine one component for each vertex operator based on the previous propositions.

Theorem 4.4. The $\overline{1}$-components of the type I vertex operator $\Phi(z)_{\lambda}^{\mu V}$ with respect to $V_{z}: V(\lambda) \longrightarrow V(\mu) \otimes V_{z}$ can be realized explicitly as follows:

$$
\begin{aligned}
\Phi_{\overline{1}}(z)= & \exp \left(\sum \frac{[k]}{k} q^{\frac{4 n+3}{2} k} a_{\overline{1}}(-k)(-z)^{k}\right) \exp \left(\sum \frac{[k]}{k} q^{-\frac{4 n+1}{2} k} a_{\overline{1}}(k)(-z)^{-k}\right) \\
& \times e^{\lambda_{1}}\left(-q^{2 n+1} z\right)^{\partial_{\lambda_{1}}+\left(\lambda_{1} \mid \lambda_{1}-\bar{\mu}\right)} b_{\lambda}^{\mu}, \text { for } A_{2 n-1}^{(2)}, \\
\Phi_{\overline{1}}(z)= & \exp \left(\sum \frac{[k]}{k} q^{(4 n+3) k}(-1)^{k n} a_{\overline{1}}(-2 k) z^{2 k}\right) \\
& \cdot \exp \left(\sum \frac{[k]}{k} q^{-(2 n+1) k}(-1)^{k n} a_{\overline{1}}(2 k) z^{-2 k}\right) \\
& \times e^{\lambda_{1}}\left(-q^{2 n+1} z\right)^{\partial_{\lambda_{1}}+\left(\lambda_{1} \mid \lambda_{1}-\bar{\mu}\right)} b_{\lambda}^{\mu}, \quad \text { for } D_{n+1}^{(2)}, \\
\Phi_{\overline{1}}(z)= & \exp \left(\sum \frac{[k]}{k} q^{\frac{4 n+5}{2} k} a_{\overline{1}}(-k)(-z)^{k}\right) \exp \left(\sum \frac{[k]}{k} q^{-\frac{4 n+3}{2} k} a_{\overline{1}}(k)(-z)^{-k}\right) \\
& \times e^{\lambda_{1}}\left(-q^{2 n+2} z\right)^{\partial_{\lambda_{1}}+\left(\lambda_{1} \mid \lambda_{1}-\bar{\mu}\right)} b_{\lambda}^{\mu}, \quad \text { for } A_{2 n}^{(2)}, \\
\Phi_{\overline{1}}(z)= & \exp \left(\sum \frac{[k]}{k} q^{\frac{15}{2} k} a_{\overline{1}}(-k) z^{k}\right) \exp \left(\sum \frac{[k]}{k} q^{-\frac{13}{2} k} a_{\overline{1}}(k) z^{-k}\right) \\
& \times e^{\lambda_{1}}\left(-q^{7} z\right)^{\partial_{\lambda_{1}}+\left(\lambda_{1} \mid \lambda_{1}-\bar{\mu}\right)} b_{\lambda}^{\mu}, \quad \text { for } D_{4}^{(3)},
\end{aligned}
$$

where $b_{\lambda}^{\mu}$ is a constant for each case $\left(b_{\Lambda_{0}}^{\mu}=1\right)$ and - is the canonical projection $\hat{P} \longrightarrow P$. by

The 1-component of type I vertex operator $\Phi_{\lambda}^{\mu V^{*}}(z)$ associated with $V_{z}^{*}$ is given

$$
\begin{aligned}
\Phi_{1}^{*}(z)= & \exp \left(\sum \frac{[k]}{k} q^{3 k / 2} a_{\overline{1}}(-k) z^{k}\right) \exp \left(\sum \frac{[k]}{k} q^{-k / 2} a_{\overline{1}}(k) z^{-k}\right) \\
& \times e^{\lambda_{1}}(-q z)^{\partial_{\lambda_{1}}+\left(\lambda_{1} \mid \lambda_{1}-\bar{\mu}\right)} b_{\lambda}^{\mu}, \quad \text { for } A_{2 n-1}^{(2)}, A_{2 n}^{(2)}, D_{4}^{(3)}, \\
\Phi_{1}^{*}(z)=\exp \left(\sum \frac{[k]}{k} q^{3 k / 2}(-1)^{k n} a_{\overline{1}}(-2 k) z^{k}\right) & \\
& \cdot \exp \left(\sum \frac{[k]}{k} q^{-k / 2}(-1)^{k n} a_{\overline{1}}(2 k) z^{-2 k}\right) \\
& \times e^{\lambda_{1}}(q z)^{\partial_{\lambda_{1}}+\left(\lambda_{1} \mid \lambda_{1}-\bar{\mu}\right)} b_{\lambda}^{\mu}, \quad \text { for } D_{n+1}^{(2)},
\end{aligned}
$$

where $b_{\lambda}^{\mu}$ is a constant for each case $\left(b_{\Lambda_{0}}^{\mu}=1\right)$.

Proof. The theorem is shown by demonstrating that the construction satisfies all the intertwining relations.

The exponential factors of Heisenberg generators are required by the commutation relations in Proposition 4.3 and the definition of $a_{\overline{1}}(k)$. The commutation relation of $\Phi(z)$ or $\Phi(z)^{*}$ with $t_{j}$ asserts the factor $e^{\lambda_{1}}$. The various factors involving $\partial_{\lambda_{1}}$ simply assure that the vertex operators commute with $X_{j}^{+}(z)$. For instance 
in the case of the type I vertex operator with respect to $V_{z}$ we have

$$
\begin{aligned}
& {\left[\Phi_{\overline{1}}(z), X_{j}^{+}(w)\right]} \\
& \quad=: \Phi_{\overline{1}}(z) X_{j}^{+}(w): \kappa(z)^{\delta_{j n}}\left(q^{2 n} z\right)^{\left(\lambda_{1} \mid \lambda_{1}-\bar{\mu}\right)}(-1)^{\left(\lambda_{n} \mid \lambda_{1}-\bar{\mu}\right)} w^{\left(\alpha_{j} \mid \alpha_{j}\right) / 2} \\
& \quad \quad \times\left(\left(q^{2 n} z-w\right)^{\delta_{j 1}} e^{\lambda_{1}} e^{\alpha_{j}}(-1)^{\delta_{j n}}-\left(w-q^{2 n} z\right)^{\delta_{j 1}} e^{\alpha_{j}} e^{\lambda_{1}}\right)\left(q^{2 n} z\right)^{\partial_{\lambda_{1}}} w^{\partial_{\alpha_{j}}}(-1)^{\partial_{\lambda_{n}}} \\
& =0
\end{aligned}
$$

where : : is the bosonic normal ordering for the Heisenberg generators.

The appearance of $\left(\alpha_{1} \mid \lambda_{1}-\bar{\mu}\right)$ and $b_{\lambda}^{\mu}$ is due to our normalization, which is illustrated in the case $V\left(\Lambda_{1}\right) \longrightarrow V\left(\Lambda_{0}\right) \otimes V_{z}$ for $A_{2 n-1}^{(2)}$ :

$$
\begin{aligned}
\Phi_{\Lambda_{1} \overline{0}}^{\Lambda_{0} V}(0)\left|\Lambda_{1}\right\rangle & =\Phi_{\overline{1}}(0) X_{1}^{-}(0) \cdots X_{n}^{-}(0) \cdots X_{1}^{-}(0) .1 \otimes e^{\lambda_{1}} \otimes 1+\cdots \\
& =b_{\lambda_{1}}^{\lambda_{0}} e^{\lambda_{1}}\left(-q^{2 n+1}\right)^{\partial_{\lambda_{1}}+\left(\lambda_{1} \mid \lambda_{1}-\lambda_{0}\right)} \cdot 1 \otimes e^{-\alpha_{1}} \cdots e^{-\alpha_{n}} \cdots e^{-\alpha_{1}} e^{\lambda_{1}} \otimes 1 \\
& =\left|\Lambda_{0}\right\rangle
\end{aligned}
$$

By the same argument, we get the realization for the vertex operators of type II.

Theorem 4.5. The 1-components of the type II vertex operator $\Phi_{\lambda}^{V \mu}(z)$ with respect to $V_{z}: V(\lambda) \longrightarrow V_{z} \otimes V(\mu)$ can be realized explicitly as follows:

$$
\begin{aligned}
\Phi_{1}(z)= & \exp \left(\sum-\frac{[k]}{k} q^{\frac{k}{2}} a_{\overline{1}}(-k) z^{k}\right) \exp \left(-\sum \frac{[k]}{k} q^{-\frac{-3 k}{2}} a_{\overline{1}}(k) z^{-k}\right) \\
& \times e^{-\lambda_{1}}(-q z)^{-\partial_{\lambda_{1}}+\left(\lambda_{1} \mid \lambda_{1}+\bar{\mu}\right)} b_{\lambda}^{\mu}, \quad \text { for } A_{2 n-1}^{(2)}, A_{2 n}^{(2)}, D_{4}^{(3)} \\
= & \exp \left(\sum-\frac{[k]}{k} q^{k}(-1)^{k n} a_{\overline{1}}(-2 k) z^{2 k}\right) \exp \left(-\sum \frac{[k]_{1}}{k} q^{-3 k} a_{\overline{1}}(2 k) z^{-2 k}\right) \\
& \times e^{-\lambda_{1}}(-q z)^{-\partial_{\lambda_{1}}+\left(\lambda_{1} \mid \lambda_{1}+\bar{\mu}\right)} b_{\lambda}^{\mu}, \quad \text { for } D_{n+1}^{(2)},
\end{aligned}
$$

where $b_{\lambda}^{\mu}$ is as above. The $\overline{1}$-component of type I vertex operator $\Phi_{\overline{1}}^{*}(z)$ associated with $V_{z}^{*}$ is given by

$$
\begin{aligned}
\Phi_{\overline{1}}^{*}(z)= & \exp \left(-\sum \frac{[k]}{k} q^{\frac{-4 n+1}{2} k} a_{\overline{1}}(-k)(-z)^{k}\right) \exp \left(-\sum \frac{[k]}{k} q^{\frac{4 n-3}{2} k} a_{\overline{1}}(k)(-z)^{-k}\right) \\
& \times e^{-\lambda_{1}}\left(q^{-2 n+2} z\right)^{-\partial_{\lambda_{1}}+\left(\lambda_{1} \mid \lambda_{1}+\bar{\mu}\right)} \bar{b}_{\lambda}^{\mu}, \quad \text { for } A_{2 n-1}^{(2)}, \\
\Phi_{\overline{1}}^{*}(z)= & \exp \left(-\sum \frac{\left[k_{1}\right]}{k}(-1)^{k n} q^{(-4 n+1) k} a_{\overline{1}}(-2 k) z^{2 k}\right) \\
& \times \exp \left(-\sum \frac{[k]}{k}(-1)^{k n} q^{(4 n-5) k} a_{\overline{1}}(k) z^{-2 k}\right) \\
& \times e^{-\lambda_{1}}\left(q^{-2 n+2} z\right)^{-\partial_{\lambda_{1}}+\left(\lambda_{1} \mid \lambda_{1}+\bar{\mu}\right)} \bar{b}_{\lambda}^{\mu}, \quad \text { for } D_{n+1}^{(2)}, \\
\Phi_{\overline{1}}^{*}(z)= & \exp \left(-\sum \frac{[k]}{k} q^{\frac{-4 n-1}{2} k} a_{\overline{1}}(-k)(-z)^{k}\right) \exp \left(-\sum \frac{[k]}{k} q^{\frac{4 n-1}{2} k} a_{\overline{1}}(k)(-z)^{-k}\right) \\
& \times e^{-\lambda_{1}}\left(q^{-2 n} z\right)^{-\partial_{\lambda_{1}}+\left(\lambda_{1} \mid \lambda_{1}+\bar{\mu}\right)} \bar{b}_{\lambda}^{\mu}, \quad \text { for } A_{2 n}^{(2)}, \\
\Phi_{\overline{1}}^{*}(z)= & \exp \left(-\sum \frac{[k]}{k} q^{\frac{-11}{2} k} a_{\overline{1}}(-k) z^{k}\right) \exp \left(-\sum \frac{[k]}{k} q^{\frac{9}{2} k} a_{\overline{1}}(k) z^{-k}\right) \\
& \times e^{-\lambda_{1}}\left(q^{-5} z\right)^{-\partial_{\lambda_{1}}+\left(\lambda_{1} \mid \lambda_{1}+\bar{\mu}\right)} \bar{b}_{\lambda}^{\mu}, \quad \text { for } D_{4}^{(3)},
\end{aligned}
$$

where $\bar{b}_{\lambda}^{\mu}$ is a constant so that $\bar{b}_{\lambda_{0}}^{\mu}=1$. 


\section{REFERENCES}

1. B. Davies, O. Foda, M. Jimbo, T. Miwa and A. Nakayashiki, Diagonalization of the XXZ Hamiltonian by vertex operators, Commun. Math. Phys. 151 (1993), 89-153. MR 94a:82018

2. E. Date, M. Jimbo, and M. Okado, Crystal base and q-vertex operators, Commun. Math. Phys. 155 (1993), 47-69. MR 94f:17012

3. V.G. Drinfeld, A new realization of Yangians and quantized affine algebras, Soviet Math. Dokl. 36 (1988), 212-216. MR 88j:17020

4. I.B. Frenkel and N. Jing, Vertex representations of quantum affine algebras, Proc. Natl. Acad. Sci. USA 85 (1988), 9373-9377. MR 90e:17028

5. I.B. Frenkel and N. Reshetikhin, Quantum affine algebras and holonomic difference equations, Commun. Math. Phys. 146 (1992), 1-60. MR 94c:17024

6. M. Idzumi, Level two irreducible representations of $U_{q}\left(\hat{s} l_{2}\right)$, Int. J. Mod. Phys. A. 9 (1994), 4449-4484. MR 95j:17029

7. M. Jimbo, K. Miki, T.Miwa and A. Nakayashiki, Correlation functions of the XXZ model for $\Delta<-1$, Phys. Lett. A 168 (1992), 256-263. MR 93m:82007

8. M. Jimbo and T. Miwa, Algebraic analysis of solvable lattice models, CBMS series 85, Amer. Math. Soc., Providence, RI, 1995. MR 96e:82037

9. N. Jing, Twisted vertex representations of quantum affine algebras, Invent. Math. 102 (1990), 663-690. MR 92a:17019

10. N. Jing, Higher level representations of the quantum affine algebra $U_{q}(\hat{s l}(2))$, J. Algebra 182 (1996), 448-468. MR 97f:17017

11. N. Jing, On Drinfeld realization of quantum affine algebras, in The Monster and Lie Algebras, ed. J. Ferrar and K. Harada, Ohio State Univ. Math. Res. Publ. 7, de Gruyter, Berlin-New York, 1998. q-alg/9610035.

12. N. Jing, S.-J. Kang, Y. Koyama, Vertex operators of quantum affine Lie algebras $U_{q}\left(D_{n}^{(1)}\right)$, Commun. Math. Phys. 174 (1995), 367-392. MR 96j:17022

13. N. Jing and K. C. Misra, Vertex operators of level one $U_{q}\left(B_{n}^{(1)}\right)$-modules, Lett. Math. Phys. 36 (1996), 127-143. MR 97b:17022

14. V.G. Kac, Infinite Dimensional Lie Algebras, 3rd ed., Cambridge University Press, Cambridge, 1990. MR 92k:17038

15. S.-J. Kang, M. Kashiwara, K.C. Misra, T. Miwa, T. Nakashima, and A. Nakayashiki , Affine crystals and vertex models, Inter. J. Mod. Phys. A 7 (1992), 449-484. MR 94a:17008

16. A. Kato, Y. Quano and J. Shiraishi, Free boson representation of q-vertex operators and their correlation functions, Commun. Math. Phys. 157 (1993), 119-137. MR 95a:81125

17. Y. Koyama, Staggered polarization of vertex models with $U_{q}(\widehat{s l}(n))$-symmetry, Commun. Math. Phys. 164 (1994), 277-291. MR 96h:17021

18. A. Matsuo, A q-deformation of Wakimoto modules, primary fields and screening operators, Commun. Math. Phys. 151 (1993), 89-153. MR 95h:17033

Department of Mathematics, North Carolina State University, Raleigh, North CarOLINA 27695

E-mail address: jing@eos.ncsu.edu

Department of Mathematics, North Carolina State University, Raleigh, North CarOLINA 27695

E-mail address: misra@math.ncsu.edu 